\title{
1 Large-scale identification of protein histidine methylation in human cells
}

2

3 Sebastian Kapell ${ }^{1}$ and Magnus E. Jakobsson ${ }^{2, *}$

4

$5 \quad{ }^{1}$ National Bioinformatics Infrastructure Sweden (NBIS), Science for Life Laboratory, Department of

6 Biochemistry and Biophysics, Stockholm University, 10691, Stockholm, Sweden

$7 \quad$ 2Department of Immunotechnology, Lund University, Medicon Village, Lund, Sweden.

8

9 *To whom correspondence may be addressed: Magnus E. Jakobsson,

10

magnus.jakobsson@immun.lth.se; Tel.: (+46) 735254700. 


\section{ABSTRACT}

12 Methylation can occur on histidine, lysine and arginine residues in proteins and often serves a regulatory

13 function. Histidine methylation has recently attracted notable attention through the discovery of the

14 human histidine methyltransferase enzymes SETD3 and METTL9. There are currently no methods to enrich histidine methylated peptides for mass spectrometry analysis and large-scale analyses of the modification are hitherto absent. In the present study we query ultra-comprehensive proteomic datasets to generate a resource of histidine methylation sites in human cells. We use this resource to explore the frequency, localization, targeted domains, protein types and sequence requirements of histidine methylation and benchmark all analyses to methylation events on lysine and arginine. Our results demonstrate that histidine methylation is widespread in human cells and tissues and that the modification is over-represented in regions of mono-spaced histidine repeats. We also report colocalization of the modification with functionally important phosphorylation sites and disease associated mutations to identify regions of likely regulatory and functional importance.

24 Taken together, we here report a system level analysis of human histidine methylation and our results 25 represent a comprehensive resource enabling targeted studies of individual histidine methylation events. 


\section{INTRODUCTION}

Methylation of histidine is a post translational modification (PTM) that was first described to occur on actin(1) and myosin(2) proteins around five decades ago. It can occur at two distinct positions denoted as 1-methyl histidine $(\mathbf{1} \mathbf{M e H})$ and 3-methyl histidine $(\mathbf{3 M e H})$ (here collectively referred to as $\mathrm{Hme})(3)$. Despite being known to the scientific community for long it has gained far less attention than the wellstudied protein methylation events on lysine and arginine(3), which are considered as key epigenetic modifications linked to chromatin compaction state and gene activity(4).

Until recently, little was known about the enzymology and significance of Hme. In 2018, SETD3 was uncovered as the first human methyltransferase enzyme targeting histidine and being responsible for the well-established methylation of Actin-H73(5). This finding were shortly thereafter independently validated and functionally shown to modulate smooth muscle contractility(6). In addition, the human METTL18 (also known as C1orf156) enzyme represents a clear homolog to the established yeast methyltransferase Hpm1 (systematic name YIL110W) targeting a RPL3-H243 in S. cerevisiae(7, 8), but its enzymatic activity remains to be validated. Finally, human METTL9 has been shown to act as an enzyme with broad specificity generating $1 \mathrm{MeH}$ in motifs composed of consecutive histidine residues spaced by small amino acids(9).

Aside from the few recent protein-centric studies focusing on individual Hme events, little is known about the abundance and function of the PTM. PTMs are most frequently studied at a large scale by affinity enrichment of modified peptides, or proteins, followed by mass spectrometry (MS)-based identification of targeted sites(10). Such approaches have been described for lysine methylation (Kme)(11, 12) and are well-established for arginine methylation (Rme)(13). For many PTMs, including Hme, there are no established affinity reagents, creating a need for innovative approaches for characterization. In such cases, querying ultra-comprehensive proteomic datasets for mass shifts corresponding to distinct modification events has recently emerged as a promising alternative(14). 
51 Studies dedicated to global characterization of $\mathrm{Hme}$ are until this date absent. To explore the PTM we 52 here mined a panel of ultra-deep human proteome datasets(15) to generate an extensive resource of $53 \mathrm{Hme}$ sites. The analysis revealed that $\mathrm{Hme}$ is widespread in human cells, and uncovers its abundance, 54 context, and function in relation to Kme and Rme. To the best of our knowledge, the present study 55 represents the first system level analysis of Hme and is to date the most comprehensive draft of the 56 human histidine methylome.

57 


\section{MATERIALS AND METHOD}

\section{Querying proteomic data for methylation events}

Publicly available comprehensive proteomic datasets (ProteomeXchange id: PXD004452) previously published by Bekker-Jensen et al(15) were downloaded from ProteomeXchange(16). The datasets were chosen based on exhaustive proteome depth, obtained through extensive off-line peptide fractionation using reverse phase chromatography at alkaline $\mathrm{pH}$ and analysis of individual fractions using fast scanning MS methods with a Q-Exactive HF mass spectrometer(15). The analyzed data corresponds to LC-MS/MS analysis of tryptic peptides from human tissue biopsies from colon, liver and prostate as well as the human cell lines A549, HCT116, HEK293, HeLa, MCF7 and SY5Y. In addition, an in-depth analysis of data derived from HeLa cell sample digested with a panel comprised of complementary proteases including trypsin, chymotrypsin, Glu-C and Lys-C was included to achieve comprehensive proteomic coverage.

All raw MS files were searched using MaxQuant(17) (version 1.6.0.17i) against a database containing the canonical isoforms of human proteins (Uniprot Complete proteome: UP_2017_04/Human/UP000005640_9606.fasta) using the default software settings with few exceptions. To reduce the search space, the number of allowed missed cleavages was restricted to one. In addition to the default variable modifications, corresponding to acetylation of protein $\mathrm{N}$-termini and oxidation of methionine, mono-methylation of lysine and arginine, di-methylation of lysine and arginine, tri-methylation of lysine as well as the custom generated modification mono-methylation of histidine were included as variable modifications.

\section{Bioinformatic analyses}

Bioinformatic analysis was performed using the Python programming language: Python Language Reference (version 3.8), available at http://www.python.org. The MaxQuant output files were processed, 
removing annotated contaminants. Modifications identified in one or more biological replicates containing a mono-, di- or trimethylated modification at an arginine, lysine or histidine were defined as a unique methylation site. If a methylated peptide matched to multiple protein entries, all proteins were categorized as methylated in the downstream analysis. To benchmark our identified sites, the publicly available resource PhosphoSitePlus (version 6.5.9.3)(18) was used as a reference. Protein localization data was derived from the SubCellularBarcode project(19) and the subcellular localization of proteins in the cell line MCF7 was chosen as surrogate dataset in order to infer localization of our identified methylated proteins. Complete predicted subcellular localization of all methylated proteins was also done using the computational algorithm BUSCA(20), allowing for protein assignment into 9 distinct subcellular compartments. Identification of methylations colocalizing with phosphorylation sites were achieved by searching curated phosphoproteomic dataset(21) for known phosphorylations at a distance of 10 amino acids, and the functional score of sites was defined as described in original publication. Annotated protein domains were accessed using the resource portal InterPro (version 82.0)(22) and individual methylation sites was mapped to the interior regions of protein domains annotated in the Pfam database (version 33.1)(23). Functional enrichment analysis was conducted using the String database (version 11.0)(24) and multiple testing was corrected for with the Bonferroni method for false discovery rate (FDR). Logo and enrichment analysis of amino acids flanking methylation sites were performed using the iceLogo server and the human precompiled Swiss-Prot peptide sequence composition as reference(25).

\section{Proteomic characterization of METTL9 knockout cells}

A HAP-1 METTL9 knockout (product number HZGHC004343c010, Horizon Genomics) and a wild type control cell line (product number C631, Horizon Genomics) were propagated and maintained in IMDM Glutamax media (Thermo Fisher Scientific) supplemented with 10\% fetal bovine serum (Thermo Fisher Scientific), as well as $100 \mathrm{U} / \mathrm{ml}$ penicillin and $100 \mathrm{U} / \mathrm{ml}$ streptomycin. The cells were lysed in a guanidine 
hydrochloride-based buffer and peptides were prepared for analysis using a $\mathrm{Q}$ Exactive HF mass spectrometer(26) as previously described(27).

107

The LC-MS analysis was performed using an EASY-nLC 1200 HPLC system (Thermo Fisher Scientific) coupled to a Q Exactive HF orbitrap instrument. For each single shot proteome analysis, $500 \mathrm{ng}$ peptide was separated using a $3 \mathrm{~h}$ chromatography gradient linearly ramped from $10 \%$ to $30 \%$ buffer B (80\% acetonitrile in $0.1 \%$ formic acid) in buffer $A(0.1 \%$ formic acid) during 170 minutes, whereafter the gradient was steeply ramped to $45 \%$ buffer B during 10 minutes, before column washing and equilibration. The mass spectrometer was set to continuously sample peptides through a Top12-based data dependent acquisition method. Target values for the full scan mass spectra were set to $3 e 6$ charges in the $\mathrm{m} / \mathrm{z}$ range $375-1500$ and a maximum injection time of $25 \mathrm{~ms}$ and a resolution of 60,000 at a $\mathrm{m} / \mathrm{z}$ of 200. Fragmentation of peptides was performed using higher energy C-trap dissociation (HCD) at a normalized collision energy of $28 \mathrm{eV}$. Fragment scans were performed at a resolution of 15,000 at a m/z 200 with a AGC target value of $1 \mathrm{e} 5$ and a maximum injection time of $22 \mathrm{~ms}$. To avoid repeated sequencing of peptides, a dynamic exclusion window was set to $30 \mathrm{~s}$.

The raw MS files were analyzed using MaxQuant (version 1.6.0.17i) with identical settings to the exploratory searches of published proteomic datasets, statistical analyses was performed using Perseus(28). First, LFQ intensities were imported from the MaxQuant output file denoted "protein groups". Common contaminants, proteins only identified as modified and proteins hitting the reverse decoy database were thereafter removed by filtering. The resulting data matrix was filtered for proteins detected in at least $70 \%$ of the replicates in one experimental condition. The data was then logtransformed and missing values were imputed from the lower tail of the abundance distribution using the default setting in Perseus(28). Proteins displaying significance differences between the conditions were identified through a Student's T-test $(p<0.05)$ with $P$-values corrected for multiple hypothesis testing using the Benjamini-Hochberg method. For cluster analysis, LFQ intensities for proteins 
129 displaying a significant difference between the conditions were z-scored and row and columns trees

130 were generated using Euclidean distance and Pearson correlation, respectively. Gene ontology analysis

131 of proteins over- and under-represented in METTL9 knockout cells, was performed using the embedded

132 function in Perseus and P-values were corrected using the Benjamini-Hochberg method. 


\section{RESULTS}

Histidine methylation (Figure 1) is a poorly characterized PTM which has recently attracted notable attention through the discovery of the human histidine methyltransferase enzymes SETD3(5, 6$)$ and METTL9(9). Large-scale Hme analysis is challenging since there are no available affinity agents to enrich peptides bearing the PTM for MS analysis. Here, we devise an alternative strategy based on mining ultra-deep human proteomic datasets(15) for the modification. This approach enables global identification of cellular Hme events and a subsequent system level analysis of the PTM.

\section{Histidine methylation is widespread in human cells}

To explore the abundance of Hme we searched ultra-comprehensive proteomic datasets derived from the commonly used human cell lines A549, HCT116, HEK293, HeLa, MCF7 and SY5Y as well as tissue biopsies from human colon, liver and prostate(15) (Figure 2A). The datasets were selected based on the expression of the recently established human HMT enzymes SETD3 and METTL9 (Supplementary Figure S1). The searches were performed using MaxQuant(17) with Hme defined as a custom PTM. To avoid misidentification of Hme sites, the isobaric PTMs mono-, di- and trimethylation of lysine (Kme1, Kme2 and Kme3; referred to as Kme) (Supplementary Figure S2A) as well as mono- and dimethylation of arginine (Rme1 and Rme2; referred to as Rme) (Supplementary Figure S2B) were defined as additional variable modifications (Figure 2A). This approach enables cellular Kme and Rme events to serve as a benchmark for the downstream Hme-centric analyses.

We anticipated that using a broad range of human cell proteome datasets would enable the identification of both general and cell specific Hme events. The exploratory searches revealed 267 and $80 \mathrm{Hme}$ sites across the cell lines and tissue biopsies, respectively (Figure 2B, Supplementary Table S1). Moreover, 
156 to define a core histidine methylome based on sites identified in $50 \%$, or more, of the cell lines and

157 tissue biopsies (Figure 2C). The core methylome includes two sites present in several actin variants, 158 and actin related proteins, corresponding to ACTA1-H103 and established SETD3-target site ACTB-

$159 \mathrm{H} 73(1,5,6)$ (Figure 2C and Supplementary Figure S3A). Moreover, the core methylome 160 encompasses APEX1-H151 (Uniprot id P27695), CPT2-H369 (P23786), EXOS7-H275 (Q15024), NP1L4-H4 (Q99733) and RBM22-H183 (Q9NW64) (Figure 2C) and the Hme sites in these non-actin 162 related proteins do not share apparent sequence homology (Supplementary Figure S3B).

A detailed inspection of tandem mass spectra for actin methylation revealed interesting features. First, fragment spectra were of high quality and unambiguously supported methylation of ACTB-H73, with high coverage of up- and down-stream b- and y-ions (Figure 2D). Tandem mass spectra from PTM bearing peptides can contain so-called immonium ions with a mass corresponding to the modified residue(29) and their identification may corroborate PTM events(30). Peptides containing unmodified histidine often yield a strong immonium ion with a mass of 110.0718 atomic mass units (amu) when fragmented(31). Strikingly, for the tryptic peptide covering ACTB-H73 we instead observed a clear peak at $124.0875 \mathrm{amu}$, corresponding to the mass of an Hme immonium ion (Figure 2D). Similarly, we detected an internal Hme site on the likely histidine methyltransferase METTL18 (METTL18-H154) and

172 the corresponding fragment spectra also contained a clear Hme fingerprint immonium ion 173 (Supplementary Figure S4). The observation of METTL18-H154 methylation is interesting as automethylation of methyltransferase enzymes is a well-established phenomenon, which has also previously been reported to occur with an amino acid specificity reflecting the enzymes physiological substrate(7). comprehensive proteomic datasets and that the PTM is widespread in human cells and tissues. 


\section{Exploring the HeLa methylome}

Intrigued by our observation that Hme is prevalent in human cells we embarked on an in-depth exploratory analysis of the PTM. For this analysis we focused on human HeLa cells, which is arguably the most used human cell line model in experimental research. We devised the now established successful approach of querying publicly available comprehensive datasets for Hme. This time, the chosen datasets(15) were specific for Hela cells and generated using a panel of complementary proteases including, chymotrypsin, Glu-C and Lys-C, aside from trypsin, allowing for unprecedented proteome depth and coverage(32).

Across the different proteases, the analysis revealed support for 2526 distinct cellular methylation events at 2241 sites (Figure 3B). The number of methylation events exceed the number of sites since several individual arginine and lysine residues were detected with varying degrees of methylation (Supplementary Table S1). Roughly $12 \%$ of methylation events correspond to Hme $(n=299)$ and the modification was less prevalent $(n=299)$ than Kme $(n=895)$ and Rme $(n=1332)$ (Figure 3B). In line with these observations, a previous study has suggested that roughly $14 \%$ of protein methylation events occurs on histidine(33).

A comparative analysis of our HeLa methylome data to publicly available PTM resources(18) suggests that the bulk of (>80\%) of $\mathrm{Hme}$ and Kme sites are not previously characterized (Figure $3 \mathrm{C}$ ). The fraction of novel sites was notably lower for Rme (Figure 3C), which can be expected as established workflows for affinity enrichment and MS characterization of the PTM exist(13). The large number of identified new Hme sites highlights that the generated dataset is suitable for an exploratory systems level analysis of the PTM.

To investigate whether Hme is scattered across the proteome, or frequently occurring on individual proteins, we first analyzed the number of Hme events per Hme protein. This analysis revealed that a 
202 single methylation event was identified for most $(>80 \%)$ Hme proteins and that no proteins were

203 identified with more than two Hme sites, and similar trends were observed for both Kme and Rme

204 (Figure 3D). Next, we explored the abundance of identified methyl proteins. Interestingly, we found that

$205 \mathrm{Hme}$, Kme and Rme were all overrepresented on abundant proteins (Figure 3E) and envision two 206 alternative explanations for this finding. First, the proteomics datasets in this study were generated using 207 data dependent acquisition MS, an approach intrinsically biased to identify abundant peptides and 208 PTMs(34). Alternatively, it has been suggested that certain methyltransferases have evolved specificity 209 towards key abundant cellular proteins to modulate key cellular functions(35). Prominent examples 210 include Kme and Rme in the core histone $\mathrm{H} 3(36)$, the key translational elongation factor eEF1A(37, 38), 211 and the molecular chaperone Hsp70(39-41) as well as Hme in actin(1, 5, 6), targets that were all 212 identified and validated in this study (Supplementary Table S1).

Next, we investigated the subcellular localization of methyl proteins. To this end, we used both experimental data based on the SubCellBarCode resource(19) as well as the predicted localization based on the BUSCA approach(20). Both methods place Hme, Kme and Rme proteins in the cytoplasm, nucleus, mitochondria and in secretory compartments, at comparable frequencies (Figure 3F and Supplementary Figure S5), suggesting that Hme, Kme and Rme are all widespread across cellular structures.

In summary, the above indicates that Hme is prevalent and present in all major cellular compartments.

\section{Domains and motifs targeted by Hme}

Intrigued by the finding of Hme events across different cellular compartments, we next used the Pfam $(23,42)$ database to explore which proteins families and domains that are targeted. Although Hme, 
enriched in different Pfam entries (Figure 4A). Reassuringly, the most strongly enriched Pfam entries include to domains where the methylations are well established to exert important function including Actin for $\mathrm{Hme}(5,6)$, RNA recognition for $\mathrm{Rme}(13)$ and core histone proteins for Kme(4) (Figure 4A). Notably, Hme was found overrepresented in Pfam entries associated with zinc binding properties (E3 Ligase, $\mathrm{CCCH}$ - zinc finger; Zinc finger $\mathrm{C} 2 \mathrm{H} 2$ type; Zinc finger $\mathrm{CCCH}$ type; Zinc finger $\mathrm{C}-\mathrm{x}-\mathrm{C}-\mathrm{x}-\mathrm{C}$ type (and similar); ZIP zinc transporter) (Figure 4A and Supplementary Figure S6).

Having established that Hme is over-represented in certain zinc binding proteins and domains, we next set out to analyze the sequence context flanking Hme sites using the iceLogo approach(25). We queried the 5 flanking amino acids for all detected Hme, Kme and Rme sites using the human precompiled Swiss-Prot peptide sequence composition as reference. This analysis revealed distinct sequence preferences for the different methylations (Figure 4B-D). Hme was overrepresented in mono-spaced repetitive histidine $(\mathrm{H})$ sequences (Figure 4B), Rme in glycine $(\mathrm{G})$ rich regions (Figure 4C), and Kme in the context of the acidic residues aspartate (D) and glutamate (E) (Figure 4D).

In summary, the above results demonstrate that Hme is over-represented in specific classes of zinc binding proteins and in the primary sequency context of consecutive mono-spaced histidine residues.

\section{Co-occurrence of methylation with phosphorylation and disease associated mutations}

242 It has been reported that methylation events can cross-talk with other PTM types such as phosphorylation, constructing a regulatory circuit known as methyl-phospho switch(43). For example, the lysine methyltransferase SET7 have been shown to regulate the stability of DNA methyltransferase1 (DNMT1)(44), a key enzyme in maintaining methylation patterns after DNA replication. DNMT1 is methylated at Lys142 and the adjacent Ser143 which can be phosphorylated by AKT1 kinase. These 
at Lys142. The consequence of the methylation is an overall decrease in abundance of the key epigenetic regulator DNMT1. In order to pinpoint specific Hme events of high functional importance we integrated a highly curated phosphoproteomic dataset into our analysis(21). We searched for phosphorylations on serine, threonine or tyrosine within a distance of 10 amino acids from a methylation event and this analysis revealed 1999 co-localizing phosphorylation sites (Supplemental Table S2). A distinct advantage of the curated phosphoproteomic dataset was that it had been evaluated using a novel machine learning model that integrated multiple features relating to conservation and structural properties of the phosphorylation site, that are indicative of functional relevance, thus providing a functional score. This allowed us to evaluate our identified methylation events based on the functional score of the phosphorylation site in close proximity (Figure 5A). We found that Hme, in addition to Kme and Rme, co-localized with phosphorylation sites with an above median functional score (Figure 5A).

In addition, we queried a publicly available database(45) for mutations in amino acid positions undergoing a methylation event in order to identify sites linked to pathological conditions(46). This analysis uncovered mutations of 212 methylation sites that are co-localizing with phosphorylations (Supplemental Table S3). Notably, the functional score of phosphorylation sites colocalizing with methylation site mutations also had an above the median functional score (Figure 5A).

Our analysis suggest that protein methylation co-localizes with functionally important phosphorylation sites, suggesting crosstalk between the PTMs. However, whether crosstalk has a relevance in relation to regulation of cellular function needs to be further experimentally validated for each individual protein.

\section{Identification of a conserved PTM hotspot in actin}


271 the phosphorylation of the adjacent ACTA1-Y71 site (Figure 5A and Supplemental Table S2), we

272 decided to do an in depth analysis of the region. Structurally, ACTA1-H75 is located in a loop which has

273 been reported to sense nucleotide binding(48) (Figure 5B). The loop is perfectly conserved between

274 humans, fly, plant, worm and yeast, emphasizing its functional importance (Figure 5C). To explore

275 whether the ATP-sensing loop is targeted by additional PTMs, we again queried the PhosphositePlus

276 database. This analysis revealed five annotated PTMs in the H75-containing nucleotide sensing loop

277 and its flanking residues (Figure 5D), corresponding to mono-methylation, ubiquitination, and

278 acetylation of K70 as well as phosphorylation of Y71 and T79. Notably, the bulk of these PTMs have

279 also been observed in mouse actin (Figure 5D), indicating that modification of this loop is evolutionary 280 conserved.

281

In summary, we observed disease associated mutations and PTMs at, and in proximity to, ACTA1-H75, 282

a Hme site previously categorized as belonging to the core Hme-ome. The findings emphasize the functional importance of the ATP-sensing loop harboring $\mathrm{H} 75$ and support a model where multiple PTMs may play a role in actin regulation.

\section{Cellular effects of METTL9 KO}

Having uncovered hundreds of cellular Hme events and explored the subcellular distribution and context of the PTM, we sought to also investigate its direct biochemical functions. Human METTL9 was very recently described as a histidine methyltransferase introducing the bulk of $1 \mathrm{MeH}$ in mammalian proteomes through methylation of histidine in the context of a $\mathrm{HxH}$ motif, where " $\mathrm{x}$ " is a preferentially small amino acid such as alanine, glycine or serine(9). The reported specificity of METTL9 corresponds perfectly to our observed over-represented motif for Hme (Figure 4B), highlighting METTL9 gene targeted cells as a suitable model for studies of cellular Hme loss. 
294 To uncover cellular processes regulated by Hme, we therefore obtained a CRISPR-mediated METTL9 295 knockout (KO) HAP-1 cell line(49) and characterized its steady-state proteome, compared to a wild type 296 (WT) control (Figure 6A). Cellular proteins were extracted and quantified using a label free MS approach

297 (Figure 6A). Principal component analysis of protein intensities revealed a clear separation of WT and $298 \mathrm{KO}$ cells in the first component (Figure 6B), indicating that the difference between the cellular conditions 299 exceed the technical experimental variation. Accordingly, hierarchical cluster analysis of proteins with a 300 significant difference (Student's T-test, $\mathrm{P}$-adj < 0.05 ) in abundance between the conditions revealed two 301 distinct clusters of over- and under-represented proteins in the KO cells (Figure 6C). To obtain insights 302 into the processes and functions affected in the METTL9 KO cells we performed gene ontology analyses 303 of the clusters. This analysis revealed vesicle and vesicle-related processes as under-represented and 304 nuclear nucleic acid-associated processes as over-represented in the KO cells (Figure 6D). This 305 suggests a complex and pleiotropic phenotype caused by METTL9 deletion and loss of pervasive Hme1. 


\section{Discussion}

307

We here report the first large-scale analysis of Hme and demonstrate that the PTM is widespread in human cells and tissues. Our analyses indicate that the PTM is present in all major cellular compartments and that it is overrepresented in specific protein families, in particular in actin and in zincbinding proteins. Taken together, we present the hitherto most extensive resource on cellular Hme events and perform the first system level analysis of the PTM.

Global PTM analysis almost invariably involves PTM affinity enrichment followed by MS analysis. Such approaches have contributed greatly to the knowledge on phosphorylation(50, 51), acetylation(52), ubiquitination(53), SUMOylation(54) and Rme(13) but it has been more challenging to generate robust affinity agents for proteomics characterization of $\mathrm{Kme}$, and to the best of our knowledge, has not yet been tried for Hme. To study Hme, we here deployed an alternative brute-force approach, taking advantage of the high throughput of modern mass spectrometers, and queried ultra-comprehensive proteomic datasets for the PTM. To provide a benchmark for identified Hme events, we also searched the datasets for Rme and Kme. The strength of our approach can be highlighted by comparing our identified Kme-ome to the current state-of-the-art Kme proteomics studies. We identified $895 \mathrm{Kme}$ events in HeLa cells alone (Figure 2B). For comparison, a study Cao et al(12) using lab-specific lgGs for all Kme states identified 552 Kme events in HeLa cells by another acknowledged study by Moore et al(11) using the bispecific Kme1 and Kme2 binding 3xMBT domain revealed $31 \mathrm{Kme}$ events in 293T cells.

A drawback with our approach is the extensive laboratory work associated with off-line fractionation of peptides before MS analysis required to generate ultra-comprehensive proteomic datasets. Moreover, searching comprehensive datasets for several variable PTMs is both computationally challenging and time consuming. The establishment of Hme affinity enrichment workflows for MS would reduce the requirements for MS analysis time and data processing, and drastically reduce the efforts and costs 
associated with our Hme-omic approach. Thus, we foresee efforts will be made towards the generation of Hme-specific affinity agents and their optimization for Hme-proteomic applications. The affinity agents can be generated though classical animal immunizations but this approach is inherently associated with low reproducibility(55). A more robust and reproducible approach would be to generate recombinant Hme-binders, using phage-displayed recombinant antibody libraries, an approach proven feasible for the PTM sulpho-tyrosine(56).

Affinity enrichment followed by MS is arguably the most widely employed approach to study PTMs. A prominent example of this is for phosphorylations, where affinity agents that specifically bind to the modified functional phosphate group exist(51). These rely on immobilized metal cations with affinity for the negatively charged phosphate group and can consequently be used for enrichment of phosphorylated serine, threonine and tyrosine(10). For protein methylations, which are small and chemically subtle PTMs, there are no such affinity agents available. Antibodies and specific methyl-state binding protein domains have instead been used to enrich Kme and Rme modified peptides for MS(1113). However, antibodies and domains often display a preference for PTMs in specific contexts. For example, several Rme antibodies have a preference for flanking residues(57) and the affinity of Kmebinding domains can be affected by neighboring PTMs, such as phosphorylation(58). Although the depth of the histidine methylome may conceivably be increased beyond this study through future affinity enrichment-based approaches, the herein identified Hme events, and the subsequent bioinformatic analysis, is not skewed or biased through context-specific affinity agents.

Our proteomic characterization of METTL9 KO cells suggests a complex phenotype with perturbations of both vesicle-associated and nuclear-linked cellular process. A recent study suggested the presence of as many as 2807 candidate METTL9 target sites $(\mathrm{HxH}, \mathrm{x}=\mathrm{A}, \mathrm{N}, \mathrm{G}, \mathrm{S}$ or $\mathrm{T})$ in the human proteome(9). 
354 for METTL9 also render our METTL9 targeted cells a poor tool to study biochemical functions of

355 individual Hme events. Instead, we suggest ectopic expression of WT and HxH-mutated METTL9 356 substrates in cells as a preferred approach.

357 Actin proteins are subject to a wide range of PTMs, many of which have determined regulatory 358 function(59). One prominent example is SETD3 mediated methylation of ACTB-H73, which corresponds 359 to ACTA1-H75, which modulates actin dynamics by accelerating the assembly of actin filaments, a 360 process preventing primary dystocia(6). Another example, is a unique multi-step N-terminal processing and modification machinery involving $\mathrm{N}$-terminal acetylation of the initiating methionine (iMet), followed by excision of iMet and subsequent acetylation of the residue in position 2(60). Our integrated analysis of Hme colocalization with machine learning predicted functional importance scored phosphorylation events (Figure 5A) uncovered ACTA1-H75 as colocalizing with ACTA1-Y71 phosphorylation, a site attributed with a high function score $(0.62)^{20}$. Interestingly, we observed five additional PTMs in the six up- and downstream residues, of these four had also been reported to occur in mouse (Figure 5D). To the best of our knowledge, so far only $\mathrm{H} 75$ methylation has been the subject of detailed biochemical studies. The multiple modifications within the loop reassembles the numerous PTMs in the flexible tail of histone $\mathrm{H} 3$, a key component of the histone code determining chromatin compaction state and gene activity. Given the extent of PTMs in the functionally important ATP-sensing loop, a similar "actin code" may exist, where multiple dynamic PTMs collectively, or individually, determine the molecular functions 372 of actin.

373 The protein methylation dataset we have generated may support further studies on Hme and we envision three direct applications. First, synthetic peptides corresponding to the relatively small HeLa Hme-ome $(n=299)$ can be generated and evaluated as substrates for new candidate histidine methyltransferase enzymes. The human genome encodes over 200 predicted methyltransferases(61) 
378 a considerable fraction of these may catalyze Hme. Candidate histidine methyltransferases may be 379 cloned, expressed, and isolated from bacterial systems, and evaluated for activity on peptide arrays 380 comprising the Hme-ome. Second, a synthetic peptide library corresponding to the Hme-ome may be 381 used to uncover Hme-driven protein interactions for yet not discovered $\mathrm{Hme}$ reader proteins and $\mathrm{Hme}$ 382 demetylases through affinity-enrichment MS approaches(27, 62). Third, our resource provides the 383 necessary information to design large-scale targeted MS methods(63) for Hme that can be used to 384 further explore the regulation and variation of Hme-ome in human cells, tissues, and biological fluids.

385 In summary, our data extends the current knowledge of $\mathrm{Hme}$ and the study represents the first system 386 level analysis of the PTM. Finally, we encourage the research community to use this resource for large387 scale targeted MS and detailed biochemical studies of individual sites to shed further light on the 388 emerging field of protein methylation biology. 


\section{DATA AVAILABILITY}

390 Available on request to Magnus.Jakobsson@Immun.LTH.se

391

392 ACKNOWLEDGEMENTS

393 We would like to thank the Proteoforms@LU mass spectrometry infrastructure research team for useful

394 discussion and manuscript input, Jesper $\mathrm{V}$. Olsen for providing early access to proteomics datasets and 395 Júlia Szántó for assistance with HAP-1 cell culture.

396

397 FUNDING

398 The Crafoord Foundation [ref 20200526]. 
399

400

401

402

403

404

405

406

407

\section{References}

1. Johnson,P., Harris, C.I. and Perry,S. V. (1967) 3-Methylhistidine in Actin and Other Muscle Proteins. Biochem. J., 105, 361-370.

2. Huszar,G. and Elzinga,M. (1972) Homologous methylated and nonmethylated histidine peptides in skeletal and cardiac myosins. J. Biol. Chem., 247, 745-753.

3. Kwiatkowski,S. and Drozak,J. (2020) Protein Histidine Methylation. Curr. Protein Pept. Sci., 21, $675-689$.

4. Strahl,B.D. and Allis,C.D. (2000) The language of covalent histone modifications. Nature, 403, 4145.

5. Kwiatkowski,S., Seliga,A.K., Vertommen,D., Terreri,M., Ishikawa,T., Grabowska,I., Tiebe,M., Teleman,A.A., Jagielski,A.K., Veiga-Da-Cunha,M., et al. (2018) SETD3 protein is the actinspecific histidine N-methyltransferase. Elife, 7, 1-42.

6. Wilkinson,A.W., Diep,J., Dai,S., Liu,S., Ooi,Y.S., Song,D., Li,T.M., Horton,J.R., Zhang,X., Liu,C., et al. (2019) SETD3 is an actin histidine methyltransferase that prevents primary dystocia. Nature, $565,372-376$.

7. Kernstock,S., Davydova,E., Jakobsson,M., Moen,A., Pettersen,S., Mælandsmo,G.M., EggeJacobsen,W. and Falnes,P.Ø. (2012) Lysine methylation of VCP by a member of a novel human protein methyltransferase family. Nat. Commun., 3, 1038.

8. Webb,K.J., Zurita-Lopez,C.I., Al-Hadid,Q., Laganowsky,A., Young,B.D., Lipson,R.S., Souda,P., Faull,K.F., Whitelegge,J.P. and Clarke,S.G. (2010) A novel 3-methylhistidine modification of yeast ribosomal protein Rpl3 is dependent upon the YIL110W methyltransferase. J. Biol. Chem., 285, 37598-37606. 


\begin{abstract}
421 9. Davydova,E., Shimazu,T., Schuhmacher,M.K., Jakobsson,M.E., Willemen,H.L.D.M., Liu,T., 422 Moen,A., Ho,A.Y.Y., Schroer,L., Pinto,R., et al. (2021) The methyltransferase METTL9 mediates 423

10. Olsen,J. V. and Mann,M. (2013) Status of Large-scale Analysis of Post-translational Modifications by Mass Spectrometry. Mol. Cell. Proteomics, 12, 3444-3452.

11. Moore,K.E., Carlson,S.M., Camp,N.D., Cheung,P., James,R.G., Chua,K.F., Wolf-yadlin,A. and Gozani,O. (2013) A General Molecular Affinity Strategy for Global Detection and Proteomic Analysis of Lysine Methylation. Mol. Cell, 50, 444-456.

12. Cao,X.J., Arnaudo,A.M. and Garcia,B.A. (2013) Large-scale global identification of protein lysine methylation in vivo. Epigenetics, 8, 477-485.

13. Larsen,S.C., Sylvestersen,K.B., Mund,A., Lyon,D., Mullari,M., Madsen,M. V., Daniel,J.A., Jensen,L.J. and Nielsen,M.L. (2016) Proteome-wide analysis of arginine monomethylation reveals widespread occurrence in human cells. Sci. Signal., 9, 1-15.

14. Devabhaktuni,A., Lin,S., Zhang,L., Swaminathan,K., Gonzalez,C.G., Olsson,N., Pearlman,S.M., Rawson,K. and Elias,J.E. (2019) TagGraph reveals vast protein modification landscapes from large tandem mass spectrometry datasets. Nat. Biotechnol., 37, 469-479.

15. Bekker-Jensen,D.B., Kelstrup,C.D., Batth,T.S., Larsen,S.C., Haldrup,C., Bramsen,J.B., Sørensen,K.D., Høyer,S., Ørntoft,T.F., Andersen,C.L., et al. (2017) An Optimized Shotgun Strategy for the Rapid Generation of Comprehensive Human Proteomes. Cell Syst., 4, 587599.e4.

16. Deutsch,E.W., Csordas,A., Sun,Z., Jarnuczak,A., Perez-Riverol,Y., Ternent,T., Campbell,D.S., 
Bernal-Llinares,M., Okuda,S., Kawano,S., et al. (2017) The ProteomeXchange consortium in 2017: Supporting the cultural change in proteomics public data deposition. Nucleic Acids Res., 45, D1100-D1106.

17. Cox,J. and Mann,M. (2008) MaxQuant enables high peptide identification rates, individualized p.p.b.-range mass accuracies and proteome-wide protein quantification. Nat. Biotechnol., 26, $1367-1372$.

18. Hornbeck,P. V., Kornhauser,J.M., Tkachev,S., Zhang,B., Skrzypek,E., Murray,B., Latham,V. and Sullivan,M. (2012) PhosphoSitePlus: A comprehensive resource for investigating the structure and function of experimentally determined post-translational modifications in man and mouse. Nucleic Acids Res., 40, 261-270.

20. Savojardo,C., Martelli,P.L., Fariselli,P., Profiti,G. and Casadio,R. (2018) BUSCA: An integrative web server to predict subcellular localization of proteins. Nucleic Acids Res., 46, W459-W466.

21. Ochoa,D., Jarnuczak,A.F., Viéitez,C., Gehre,M., Soucheray,M., Mateus,A., Kleefeldt,A.A., Hill,A., Garcia-Alonso,L., Stein,F., et al. (2020) The functional landscape of the human phosphoproteome. Nat. Biotechnol., 38, 365-373.

22. Hunter,S., Apweiler,R., Attwood,T.K., Bairoch,A., Bateman,A., Binns,D., Bork,P., Das,U., Daugherty,L., Duquenne,L., et al. (2009) InterPro: The integrative protein signature database. Nucleic Acids Res., 37, 211-215.

23. Bateman,A., Coin,L., Durbin,R., Finn,R.D., Hollich,V., Griffiths-Jones,S., Khanna,A., Marshall,M., 

Moxon,S., Sonnhammer,E.L.L., et al. (2004) The Pfam protein families database. Nucleic Acids Res., 32, 138-141.

24. Szklarczyk,D., Gable,A.L., Lyon,D., Junge,A., Wyder,S., Huerta-Cepas,J., Simonovic,M., Doncheva,N.T., Morris,J.H., Bork,P., et al. (2019) STRING v11: Protein-protein association networks with increased coverage, supporting functional discovery in genome-wide experimental datasets. Nucleic Acids Res., 47, D607-D613.

25. Colaert,N., Helsens,K., Martens,L., Vandekerckhove,J. and Gevaert,K. (2009) Improved visualization of protein consensus sequences by iceLogo. Nat. Methods, 6, 786-787.

26. Kelstrup,C.D., Jersie-Christensen,R.R., Batth,T.S., Arrey,T.N., Kuehn,A., Kellmann,M. and Olsen,J. V. (2014) Rapid and deep proteomes by faster sequencing on a benchtop quadrupole ultra-high-field orbitrap mass spectrometer. J. Proteome Res., 13, 6187-6195.

27. Jakobsson,M.E., Małecki,J.M., Halabelian,L., Nilges,B.S., Pinto,R., Kudithipudi,S., Munk,S., Davydova,E., Zuhairi,F.R., Arrowsmith,C.H., et al. (2018) The dual methyltransferase METTL13 targets $\mathrm{N}$ terminus and Lys55 of eEF1A and modulates codon-specific translation rates. Nat. Commun., 9, 3411.

28. Tyanova,S., Temu,T., Sinitcyn,P., Carlson,A., Hein,M.Y., Geiger,T., Mann,M. and Cox,J. (2016) The Perseus computational platform for comprehensive analysis of (prote)omics data. Nat. Methods, 13, 731-740.

29. Potel,C.M., Lin,M.H., Prust,N., Van Den Toorn,H.W.P., Heck,A.J.R. and Lemeer,S. (2019) Gaining Confidence in the Elusive Histidine Phosphoproteome. Anal. Chem., 91, 5542-5547.

30. Trelle,M.B. and Jensen,O.N. (2008) Utility of immonium ions for assignment of $\varepsilon$-N-acetyllysinecontaining peptides by tandem mass spectrometry. Anal. Chem., 80, 3422-3430. 
31. Steen,H. and Mann,M. (2004) The ABC's (and XYZ's) of peptide sequencing. Nat. Rev. Mol. Cell Biol., 5, 699-711.

32. Ly,T. and Lamond,A.I. (2017) New Apex in Proteome Analysis. Cell Syst., 4, 581-582.

33. Ning,Z., Star,A.T., Mierzwa,A., Lanouette,S., Mayne,J., Couture,J.F. and Figeys,D. (2016) A charge-suppressing strategy for probing protein methylation. Chem. Commun., 52, 5474-5477.

34. Bantscheff,M., Schirle,M., Sweetman,G., Rick,J. and Kuster,B. (2007) Quantitative mass spectrometry in proteomics: A critical review. Anal. Bioanal. Chem., 389, 1017-1031.

35. Falnes,P.Ø., Jakobsson,M.E., Davydova,E., Ho,A. and Małecki,J. (2016) Protein lysine methylation by seven- $\beta$-strand methyltransferases. Biochem. J., 473, 1995-2009.

36. Turner,B.M. (2002) Cellular memory and the histone code. Cell, 111, 285-291.

37. Jakobsson,M.E., Małecki,J., Nilges,B.S., Moen,A., Leidel,S.A. and Falnes,P.Ø. (2017) Methylation of human eukaryotic elongation factor alpha (eEF1A) by a member of a novel protein lysine methyltransferase family modulates mRNA translation. Nucleic Acids Res., 45, 8239-8254.

38. Jakobsson,M.E., Małecki,J. and Falnes,P.Ø. (2018) Regulation of eukaryotic elongation factor 1 alpha (eEF1A) by dynamic lysine methylation. RNA Biol., 6286, 01-11.

39. Jakobsson,M.E., Moen,A., Bousset,L., Egge-Jacobsen,W., Kernstock,S., Melki,R. and Falnes,P. (2013) Identification and characterization of a novel human methyltransferase modulating Hsp70 protein function through lysine methylation. J. Biol. Chem., 288, 27752-27763.

40. Jakobsson,M.E., Moen,A. and Falnes,P.O. (2016) Correspondence: On the enzymology and significance of HSPA1 lysine methylation. Nat Commun, 7, 11464.

41. Szewczyk,M.M., Ishikawa,Y., Organ,S., Sakai,N., Li,F., Halabelian,L., Ackloo,S., Couzens,A.L., 
Eram,M., Dilworth,D., et al. (2020) Pharmacological inhibition of PRMT7 links arginine monomethylation to the cellular stress response. Nat. Commun., 11, 1-15.

510

511

512

513

514

515

516

517

518

519

520

521

522

523

524

525

526

527

528

529

42. Sonnhammer,E.L.L., Eddy,S.R., Birney,E., Bateman,A. and Durbin,R. (1998) Pfam: Multiple sequence alignments and HMM-profiles of protein domains. Nucleic Acids Res., 26, 320-322.

43. Sabbattini,P., Sjoberg,M., Nikic,S., Frangini,A., Holmqvist,P.H., Kunowska,N., Carroll,T., Brookes,E., Arthur,S.J., Pombo,A., et al. (2014) An H3K9/S10 methyl-phospho switch modulates Polycomb and Pol II binding at repressed genes during differentiation. Mol. Biol. Cell, 25, 904915.

44. Estéve,P.O., Chang,Y., Samaranayake,M., Upadhyay,A.K., Horton,J.R., Feehery,G.R., Cheng,X. and Pradhan,S. (2011) A methylation and phosphorylation switch between an adjacent lysine and serine determines human DNMT1 stability. Nat. Struct. Mol. Biol., 18, 42-49.

45. Liu,X., Wu,C., Li,C. and Boerwinkle,E. (2016) dbNSFP v3.0: A One-Stop Database of Functional Predictions and Annotations for Human Nonsynonymous and Splice-Site SNVs. Hum. Mutat., 37, 235-241.

46. Landrum,M.J., Lee,J.M., Riley,G.R., Jang,W., Rubinstein,W.S., Church,D.M. and Maglott,D.R. (2014) ClinVar: Public archive of relationships among sequence variation and human phenotype. Nucleic Acids Res., 42, 980-985.

47. Garcia-Angarita,N., Kirschner,J., Heiliger,M., Thirion,C., Walter,M.C., Schnittfeld-Acarlioglu,S., Albrecht,M., Müller,K., Wieczorek,D., Lochmüller,H., et al. (2009) Severe nemaline myopathy associated with consecutive mutations E74D and H75Y on a single ACTA1 allele. Neuromuscul. Disord., 19, 481-484.

48. Dominguez,R. and Holmes,K.C. (2011) Actin structure and function. Annu. Rev. Biophys., 40, 
169-186.

531

532

533

534

535

536

537

538

539

540

541

542

543

544

545

546

547

548

549

550

551

49. Essletzbichler,P., Konopka,T., Santoro,F., Chen,D., Gapp,B. V., Kralovics,R., Brummelkamp,T.R., Nijman,S.M.B. and Bürckstümmer,T. (2014) Megabase-scale deletion using CRISPR/Cas9 to generate a fully haploid human cell line. Genome Res., 24, 2059-2065.

50. Olsen,J. V, Vermeulen,M., Santamaria,A., Kumar,C., Miller,M.L., Jensen,L.J., Gnad,F., Cox,J., Jensen,T.S., Nigg,E.A., et al. (2010) Quantitative phosphoproteomics reveals widespread full phosphorylation site occupancy during mitosis. Sci. Signal., 3, ra3.

51. Olsen,J. V, Blagoev,B., Gnad,F., Macek,B., Kumar,C., Mortensen,P. and Mann,M. (2006) Global, In Vivo, and Site-Specific Phosphorylation Dynamics in Signaling Networks. Cell, 127, 635-648.

52. Choudhary,C., Kumar,C., Gnad,F., Nielsen,M.L., Rehman,M., Walther,T.C., Olsen,J. V and Mann,M. (2009) Lysine acetylation targets protein complexes and co-regulates major cellular functions. Science (80-. )., 325, 834-40.

53. Akimov,V., Barrio-Hernandez,I., Hansen,S.V.F., Hallenborg,P., Pedersen,A.K., BekkerJensen,D.B., Puglia,M., Christensen,S.D.K., Vanselow,J.T., Nielsen,M.M., et al. (2018) Ubisite approach for comprehensive mapping of lysine and n-terminal ubiquitination sites. Nat. Struct. Mol. Biol., 25.

54. Hendriks,I.A., Lyon,D., Young,C., Jensen,L.J., Vertegaal,A.C.O. and Nielsen,M.L. (2017) Sitespecific mapping of the human SUMO proteome reveals co-modification with phosphorylation. Nat. Struct. Mol. Biol., 24, 325-336.

55. Gray,A., Bradbury,A.R.M., Knappik,A., Plückthun,A., Borrebaeck,C.A.K. and Dübel,S. (2020) Animal-free alternatives and the antibody iceberg. Nat. Biotechnol., 38, 1234-1239.

56. Kehoe,J.W., Velappan,N., Walbolt,M., Rasmussen,J., King,D., Lou,J., Knopp,K., Pavlik,P., 
Marks,J.D., Bertozzi,C.R., et al. (2006) Using Phage Display to Select Antibodies Recognizing Post-translational Modifications Independently of Sequence Context. Mol. Cell. Proteomics, 5, 2350-2363.

57. Gayatri,S., Cowles,M.W., Vemulapalli,V., Cheng,D., Sun,Z.W. and Bedford,M.T. (2016) Using oriented peptide array libraries to evaluate methylarginine-specific antibodies and arginine methyltransferase substrate motifs. Sci. Rep., 6, 1-8.

58. Bock,I., Kudithipudi,S., Tamas,R., Kungulovski,G., Dhayalan,A. and Jeltsch,A. (2011) Application of Celluspots peptide arrays for the analysis of the binding specificity of epigenetic reading domains to modified histone tails. BMC Biochem., 12, 48.

59. Varland,S., Vandekerckhove,J. and Drazic,A. (2019) Actin Post-translational Modifications: The Cinderella of Cytoskeletal Control. Trends Biochem. Sci., 44, 502-516.

60. Aksnes,H., Ree,R. and Arnesen,T. (2019) Co-translational, Post-translational, and Non-catalytic Roles of N-Terminal Acetyltransferases. Mol. Cell, 73, 1097-1114.

61. Petrossian,T.C. and Clarke,S.G. (2011) Uncovering the human methyltransferasome. Mol. Cell. Proteomics, 10, M110.000976.

62. Meyer,K. and Selbach,M. (2020) Peptide-based interaction proteomics. Mol. Cell. Proteomics, 19, 1070-1075.

63. Peterson,A.C., Russell,J.D., Bailey,D.J., Westphall,M.S. and Coon,J.J. (2012) Parallel reaction monitoring for high resolution and high mass accuracy quantitative, targeted proteomics. Mol. Cell. Proteomics, 11, 1475-1488.

64. Perez-Riverol,Y., Csordas,A., Bai,J., Bernal-Llinares,M., Hewapathirana,S., Kundu,D.J., Inuganti,A., Griss,J., Mayer,G., Eisenacher,M., et al. (2019) The PRIDE database and related 
bioRxiv preprint doi: https://doi.org/10.1101/2021.03.31.437816; this version posted March 31, 2021. The copyright holder for this preprint (which was not certified by peer review) is the author/funder. All rights reserved. No reuse allowed without permission.

tools and resources in 2019: Improving support for quantification data. Nucleic Acids Res., 47, 


\section{FIGURES}

578

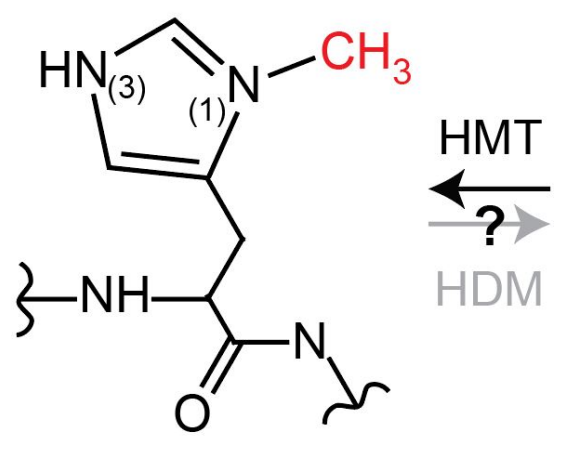

1-methyl histidine $(1 \mathrm{MeH})$

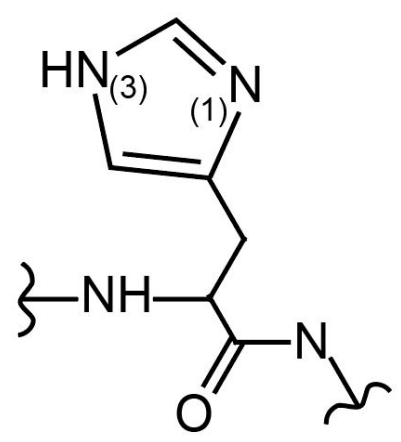

Histidine

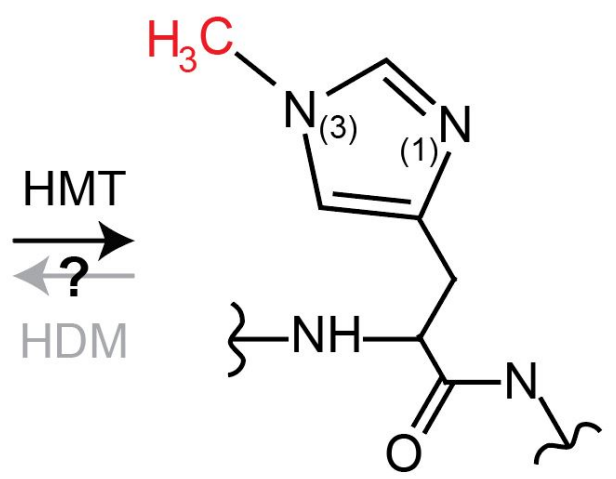

3-methyl histidine $(3 \mathrm{MeH})$

580 Figure 1. Biochemistry of protein histidine methylation.

581 Structure of the different methylated forms of histidine. The methylations are enzymatically introduced

582 by histidine $(\underline{H})$ methyltransferases $(\mathrm{HMT})$ and may potentially be removed by histidine $(\underline{\mathrm{H}})$ 583 demethylases (KDM). 

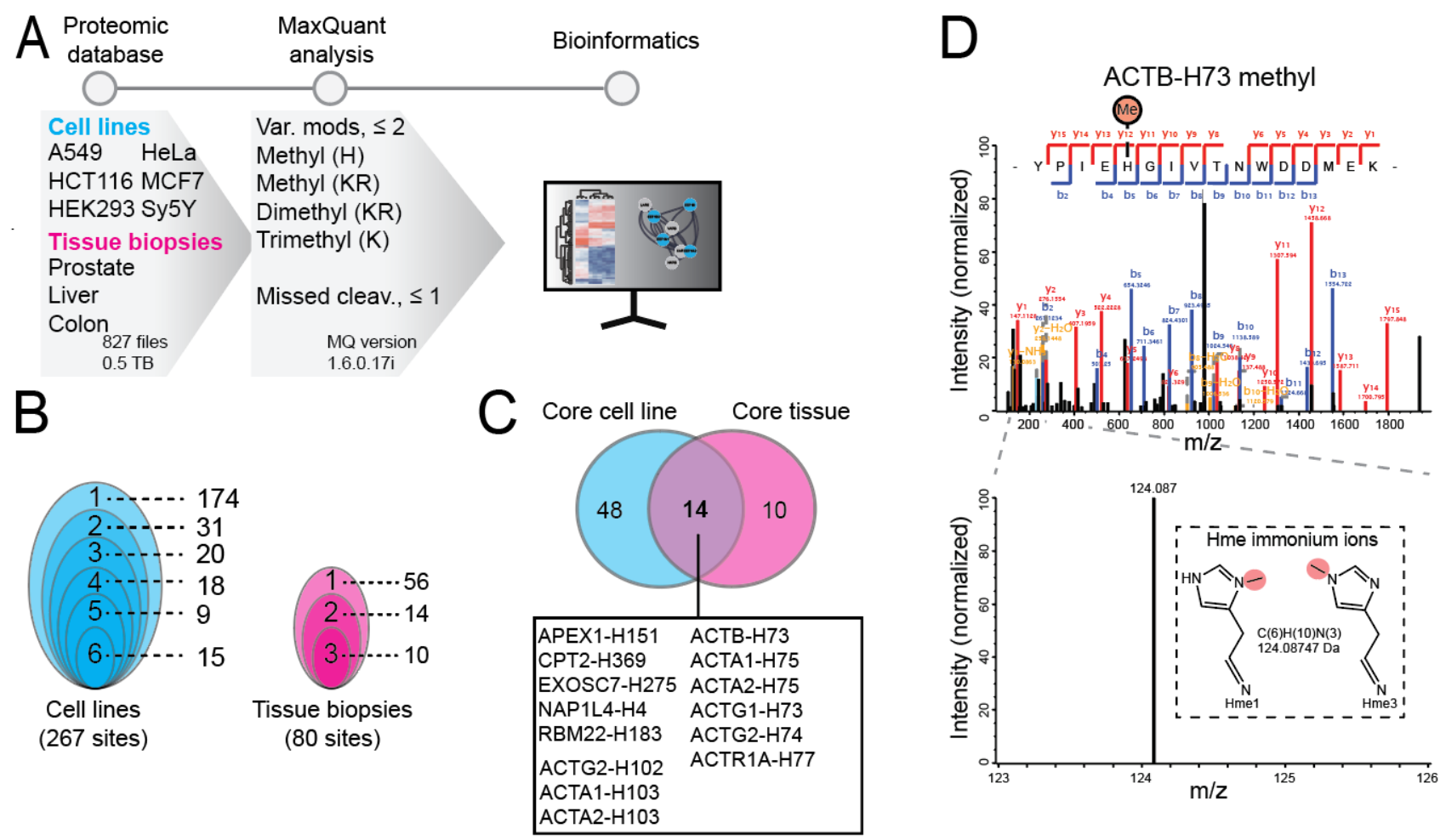

Figure 2. Exploring histidine methylation in human cells and tissues.

(A) Methylome profiling workflow. Publicly available ultra-comprehensive proteomics datasets from context of the PTM.

(B) Clam plot representation of histidine methylation sites identified in cells and tissues. The total number of sites and the number of shared sites between cell lines (left) and tissue biopsies (right) are 592 indicated. 
595 (D) Mass spectra supporting methylation of ACTB-H73. Tandem mass for a tryptic peptide covering 596 Y69-K84 of ACTB unambiguously supporting methylation of H73. The presence of a specific immonium 597 ion corresponding to methyl-histidine is indicated. 

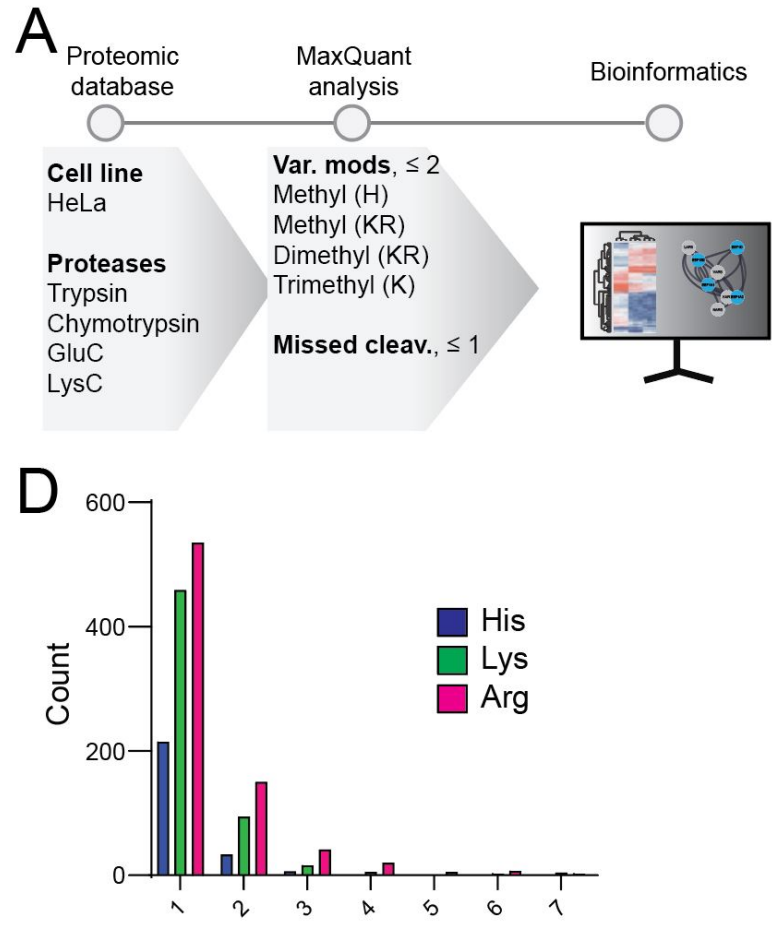

Number of methylation events per protein

$$
\text { F }
$$
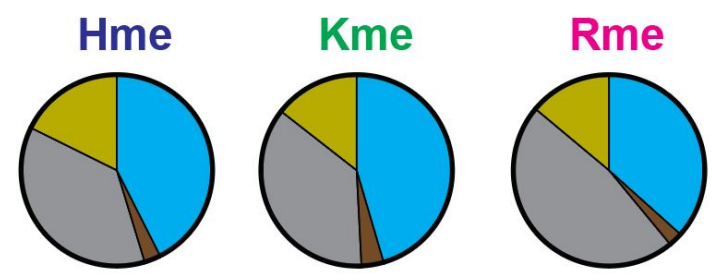

Cytoplasm

Nucleus
B

$\begin{array}{llll} & \text { Hme } & \text { Kme } & \text { Rme } \\ \text { Events } & 299 & 895 & 1332 \\ \text { Sites } & 299 & 788 & 1154 \\ \text { Proteins } & 254 & 584 & 764\end{array}$

G

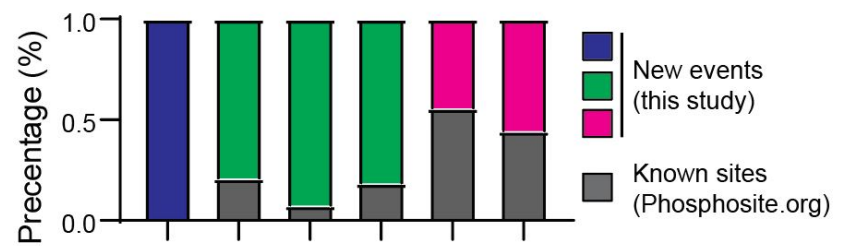

$E$

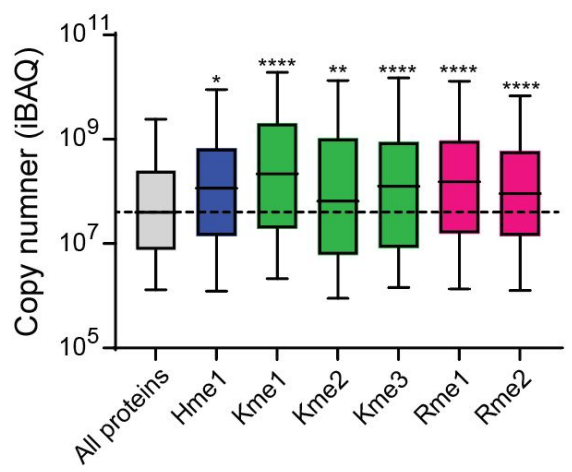

Figure 3. In-depth characterization of the HeLa methylome.

600

(A) HeLa-specific methylome profiling workflow. A panel of comprehensive proteomic datasets generated using several proteases to obtain extensive proteome and PTM coverage were searched for histidine, lysine and arginine methylation. The identified histidine methylation events were explored using a range of bioinformatic tools and benchmarked to identified lysine and arginine methylation.

(B) Total counts of distinct methylation events, methylation sites and targeted proteins are indicated. 
605 (C) The percentage of sites identified in this study as compared to the dataset available from 606 PhosphoSitePlus.

607 (D) The number of methylation events per protein.

608 (E) Cellular abundance of methyl proteins. The distribution of $i B A Q$ values for proteins harboring a 609 methylation site is shown. Significance was assessed for each group compared to control (All proteins) 610 by multiple comparison using one-way ANOVA, (adjusted $P$ value $<0.01$ ).

611 (F) Subcellular localization of proteins for Hme, Kme, Rme assigned to a designated compartment 612 neighborhood as described in the SubCellBarcode project. Each methylation type as a relative 613 distribution in the nucleus, cytoplasm, mitochondria and secretory compartment. 

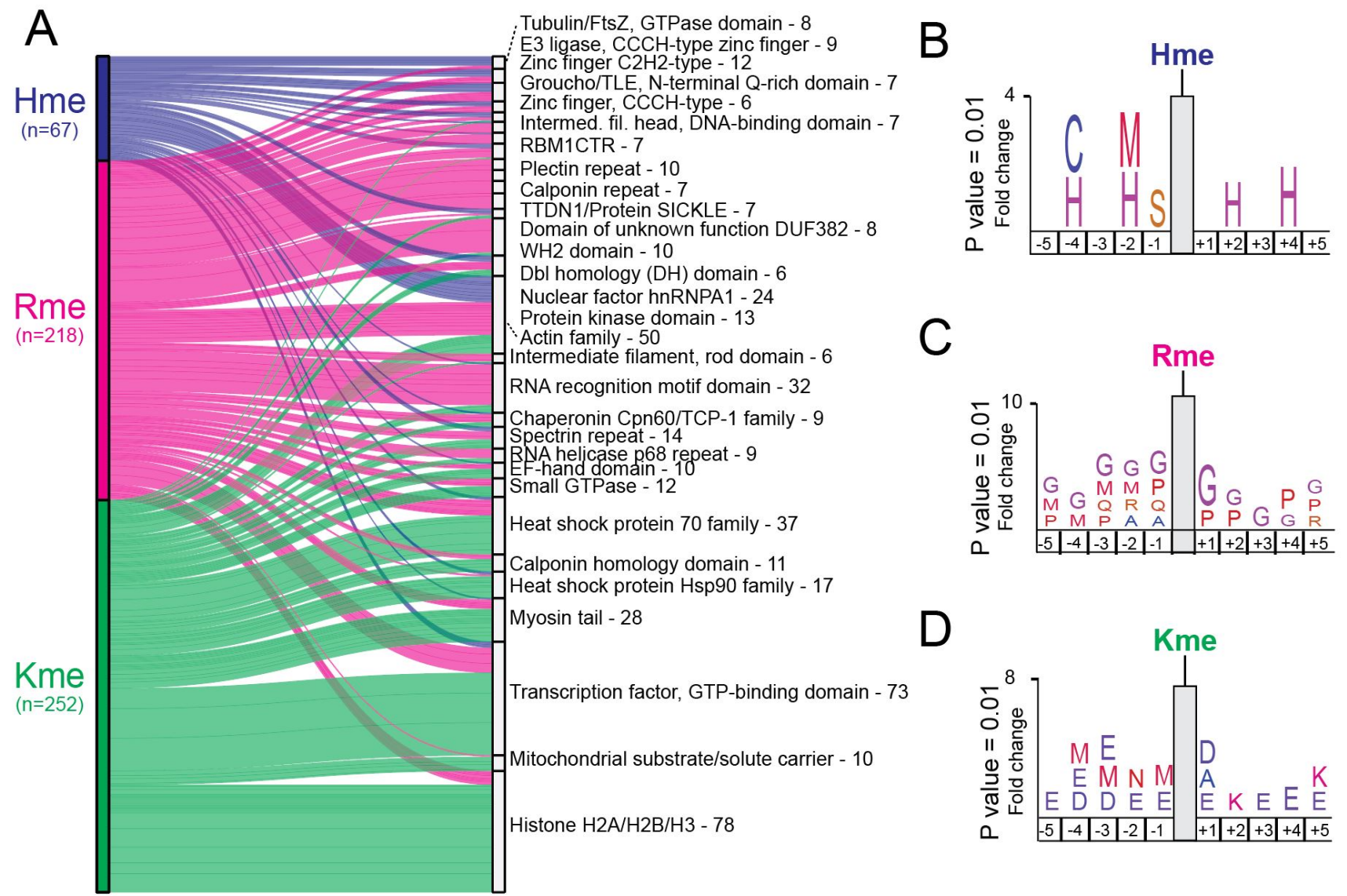

615 Figure 4. Domains and sites targeted by methylation.

616 (A) Sankey plot illustrating the top 30 Pfam domains targeted by histidine, arginine and lysine 617 methylation in HeLa cells. Total number of unique methylation sites residing within an annotated protein 618 domain in the Pfam database is indicated. Statistical analysis for significantly enriched domains is shown 619 in Supplementary Figure S6.

620 (B-D) Methylome sequence logos. Logos illustrating over representation of amino acids in the 5 621 positions up- and down-stream of identified (B) Hme, (C) Rme, and (D) Kme sites. Full sequence logos and heatmap analysis are shown in Supplementary Figure S7. 

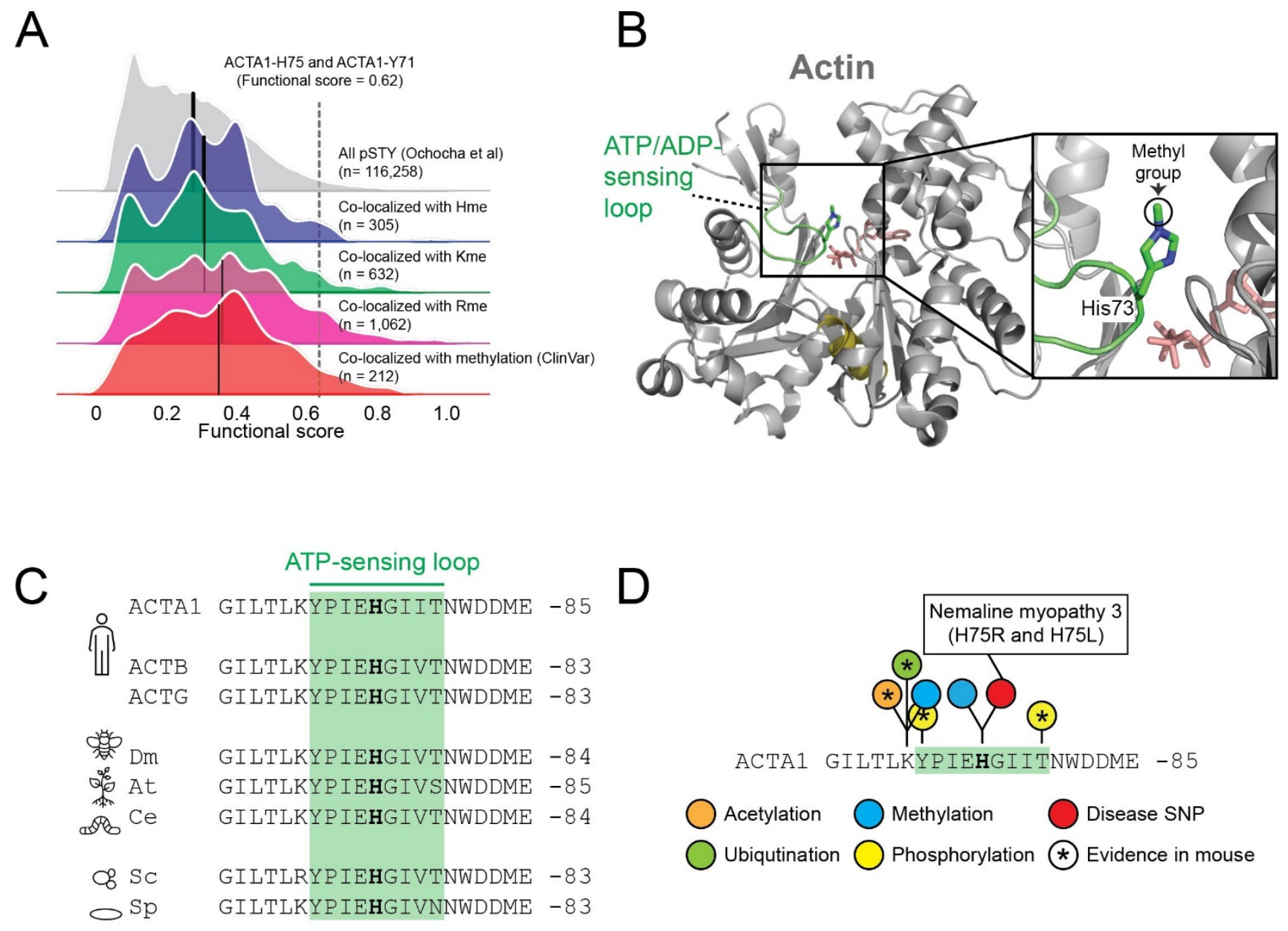

Figure 5. PTM colocalization.

625 (A) Kernel density plots for the functional score distributions of colocalizing phosphorylation sites with 626 identified methylation events. Subsets of phosphorylation sites colocalizing with Rme, Kme or Hme methylation sites plotted separately. Separate grouping of phosphorylation sites co-localizing with methylation sites with a reported polymorphism or mutation associated with a pathological condition 629 (ClinVar) is shown. Black line indicate group mean. Methylation of ACTA1-H75 and the colocalizing 630 phosphorylation event on ACTA1-Y71 are indicated.

631 (B) The structural context of actin histidine methylation. The structure of actin is shown in cartoon 632 representation whereas ATP and the methylated histidine residue $\mathrm{H} 73$ is shown in stick representation. 


\begin{abstract}
633 The hinge region (olive), ATP (salmon) and the H73 containing ATP-sensing loop (green) are indicated. 634 The structure is derived from rabbit muscle alpha actin (PDB \#1EQYE).

635 (C) Evolutionary conservation of the methyl-histidine containing loop in actin. Sequences: human 636 ACTA1 (P68133), ACTB (P60709) and ACTG (P63261) as well as homologues from Drosophila 637 melanogaster (dm; AAA28314.1), Arabidopsis thaliana (at; NP_187818.1), Caenorhabditis elegans (ce; 638 NP_508841.1), Saccharomyces cerevisiae (cs; NP_116614.1) and Saccharomyces pombe (sp; 639 NP_595618.1).
\end{abstract}

640 (D) The methyl-histidine containing loop in ACTA1 is a PTM hotspot. PTMs annotated in the 641 PhosphoSitePlus database (v6.5.9.3) are shown. Modifications observed in mouse ACTA1 (star) and 642 sites corresponding to disease associate mutations are indicated (red). 
A
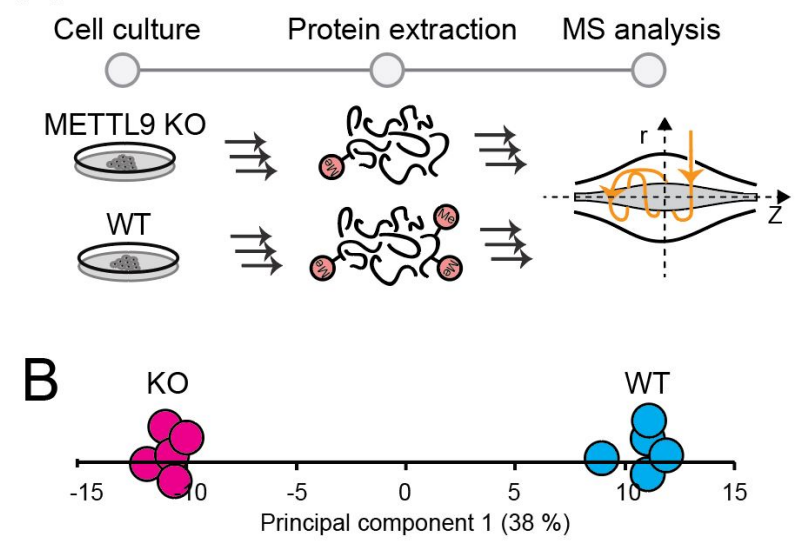

D

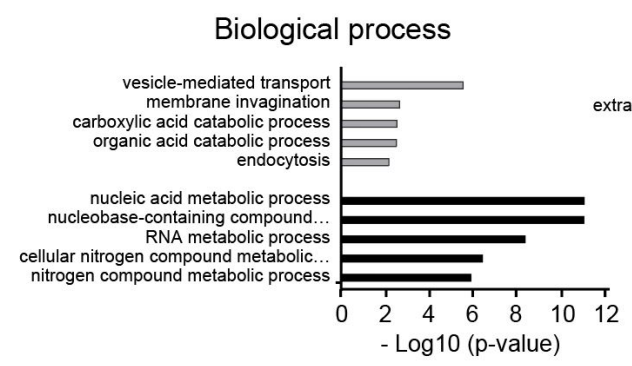

C

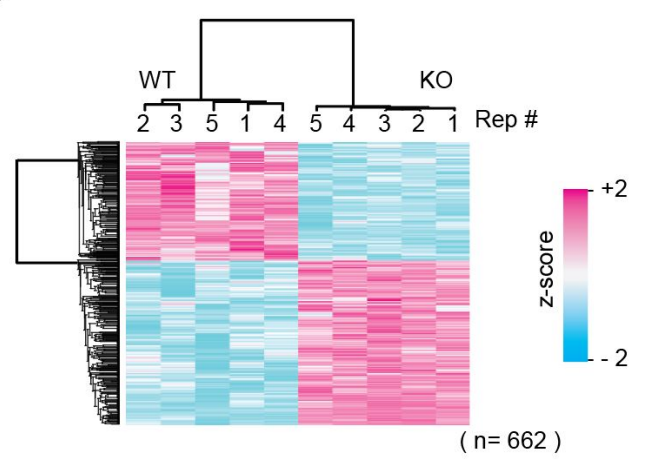

Cellular component

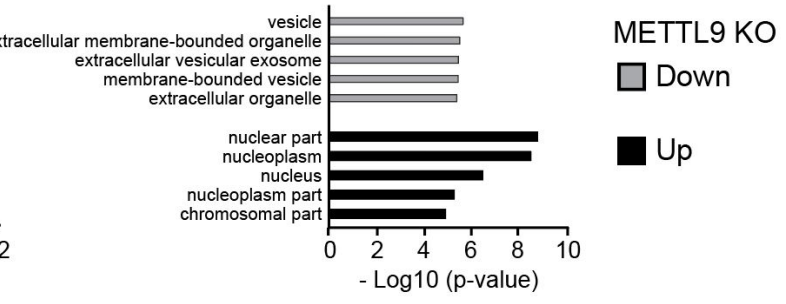

643

644 Figure 6. Cellular effects of METTL9 knockout.

645 (A) Workflow for proteomics characterization of METTL9 KO cells. Proteins were extracted from HAP-

6461 wild type (WT) and METTL9 knockout (KO) cells and processed for label free quantitative mass 647 spectrometry.

648 (B) Principal component analysis. Separation of experimental conditions (WT and KO) and replicates $649(n=5)$ in the first principal component is shown.

650 (C) Clustering analysis. Hierarchical cluster of z-scored LFQ intensities for proteins having a significant 651 difference in abundance (Student's T-test, P-adj < 0.05; Benjamini-Hochberg FDR) between the WT 652 and $\mathrm{KO}$ condition.

653 (D) Gene ontology analysis. The top five ontologies within biological process and cellular component are shown both for up- and down regulated proteins in METTL9 KO cells, relative to a WT control. 


\section{Supplementary Figure S1}
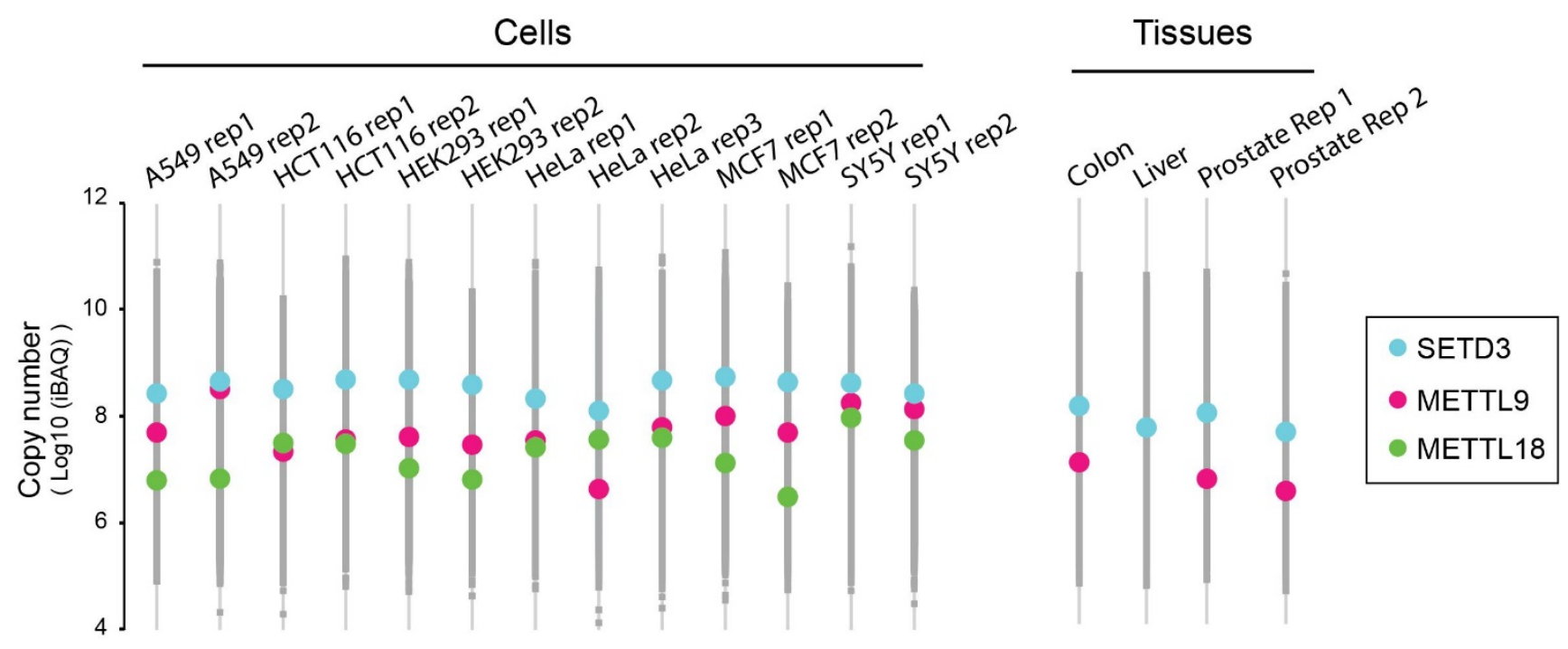

659 Supplementary Figure S1. Expression of histidine methyltransferases in cells and tissues.

660 Profile plot showing the cellular copy number (iBAQ value) for the established human histidine

661 methyltransferase enzymes SETD3 and METTL9 as well as the candidate histidine methyltransferase

662 METTL18. 


\section{A}

\section{Supplementary Figure S2}

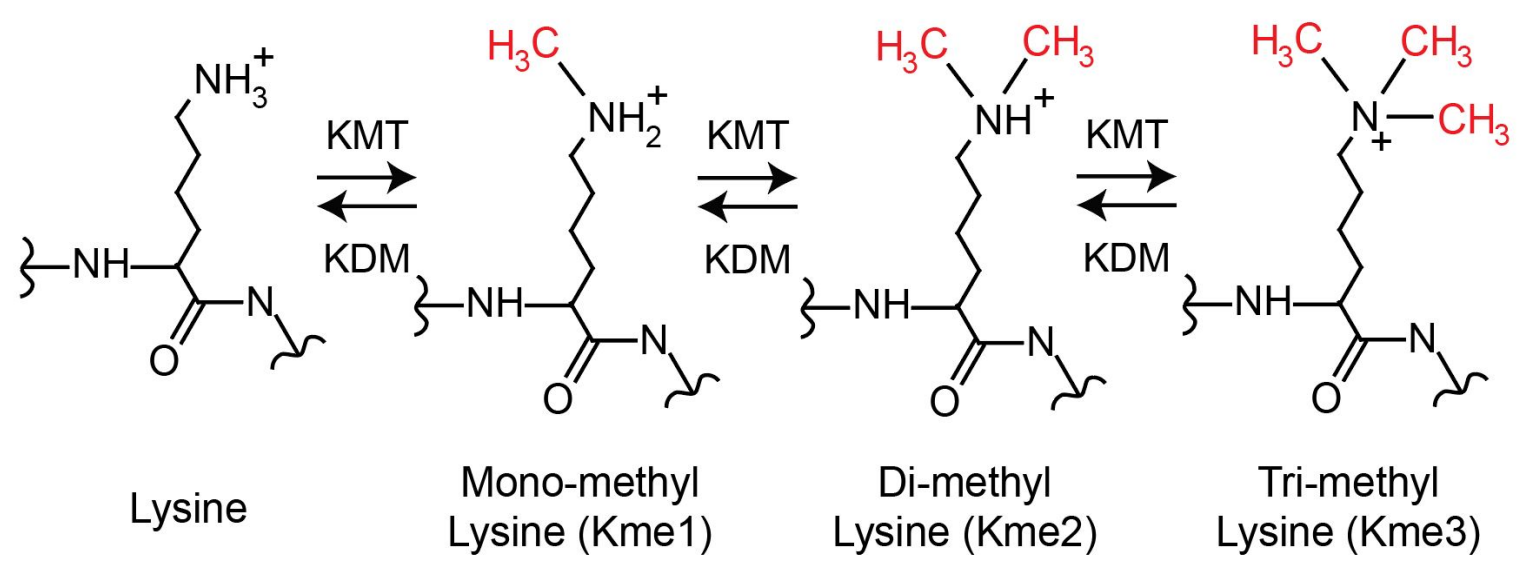

B

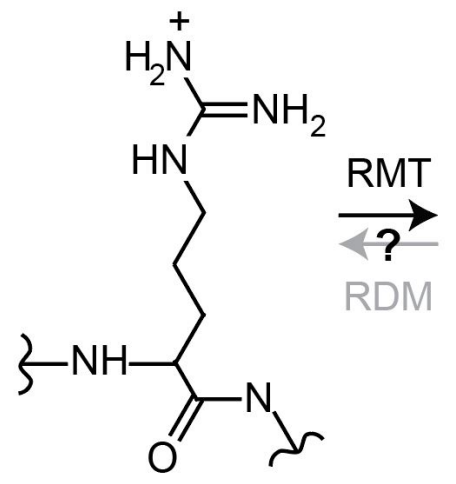

Arginine

\section{4}

665

666

667

668 669
Mono-methyl Arginine (Rme1)

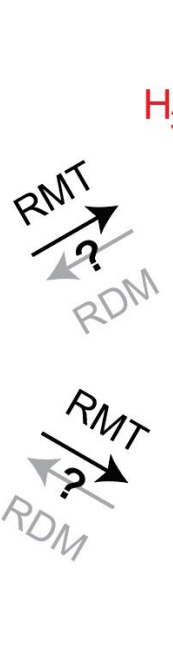

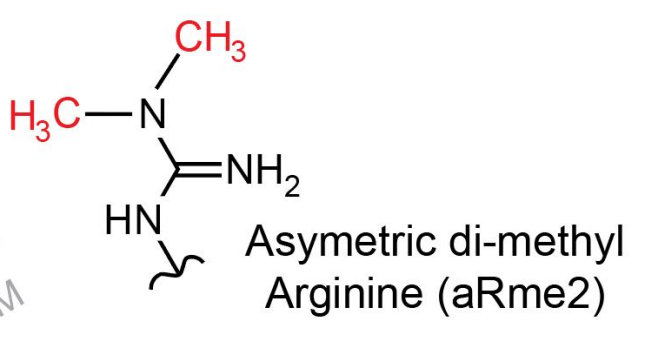

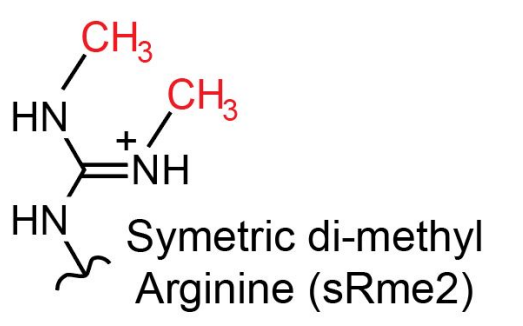

\section{Supplementary Figure S2. Biochemistry of lysine and arginine methylation.}

(A-B) The chemical structures and enzymology for $(\mathbf{A})$ lysine methylation and $(\mathbf{B})$ arginine methylation are shown. The methylations are introduced by lysine methyltransferase (KMT) and arginine methyltransferase (RMT) enzymes, respectively. Lysine methylation can be reversed by lysine demethylases (KDM). It is hypothesized, but not yet established that arginine demethylase (RDM) enzymes exist. 


\section{Supplementary Figure S3}

A

$$
\text { ACTB (P60709) }
$$

ACTG1 (P63261)

ACTA1 (P68133)

ACTA2 (P62736)

ACTG2 (P63267)

ACTR1A (P61163)

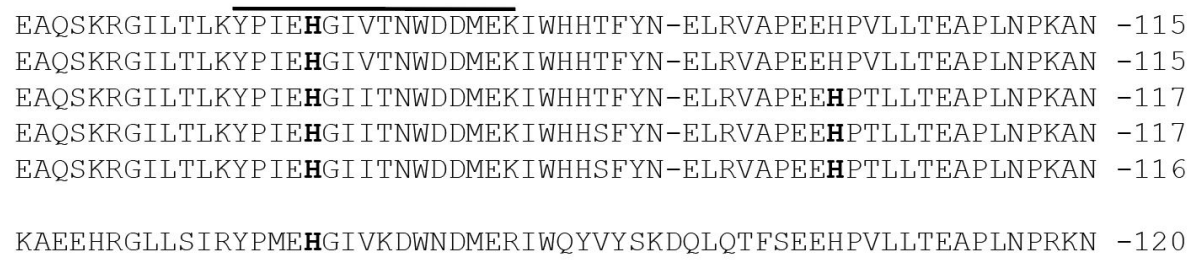

EAQSKRGILTLKYPIEHGI ITNWDDMEKIWHHTFYN-ELRVAPEEHPTLLTEAPLNPKAN -117

EAQSKRGILTLKYP IEHGIITNWDDMEKIWHHSFYN-ELRVAPEEHPTLLTEAPLNPKAN -117

KAEEHRGLLSIRYPMEHGIVKDWNDMERIWQYVYSKDQLQTFSEEHPVLLTEAPLNPRKN -120

B
APEX1 (P27695) KVSYGIGDEEHDQEGRVIVAE
CPT2(P23786) IIAKDGSTAVHFEHSWGDGVA
$-161$
EXOS7 (Q15024) VLHASLQSVVHKEESLGPKRQ
$-379$
NP1L4 (Q99733) -------MADHSFSDGVPSDS
$-285$
RBM22 (Q9NW64) CKRGEECPYRHEKPTDPDDPL
$-14$
$-193$

671 Supplementary Figure S3. Sequence alignments of core histidine methylome proteins.

672 (A) Protein sequence alignment of human actin proteins. Sites of which methylation is supported in our 673 analysis are indicated in bold.

674 (B) Protein sequence alignment of non-actin core histidine methylome proteins. Proteins shown are 675 APEX1-H151 (Uniprot id P27695), CPT2-H369 (P23786), EXOS7-H275 (Q15024), NP1L4-H4 676 (Q99733) and RBM22-H183 (Q9NW64). The histidine detected as methylated is highlighted in bold. 


\section{Supplementary Figure S4}
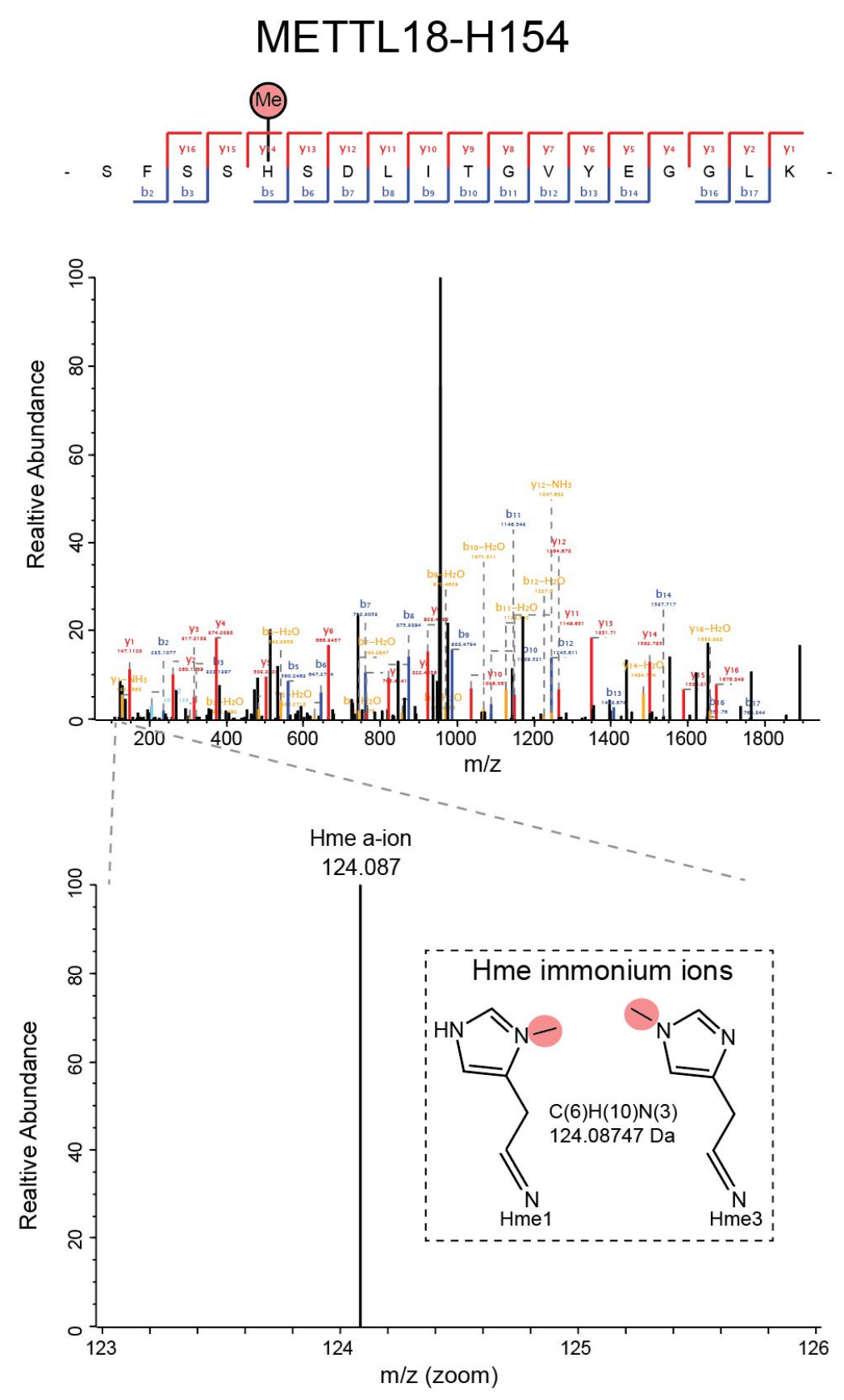

677

678 Supplementary Figure S4. METTL18 is methylated at H154 in HeLa cells.

679 Tandem mass spectrum demonstrating monomethylation of H154 in METTL18 is shown. The peptide 680 corresponds to residues S150-K168 in METTL18 (Uniprot id: O95568). 


\section{Supplementary Figure S5}
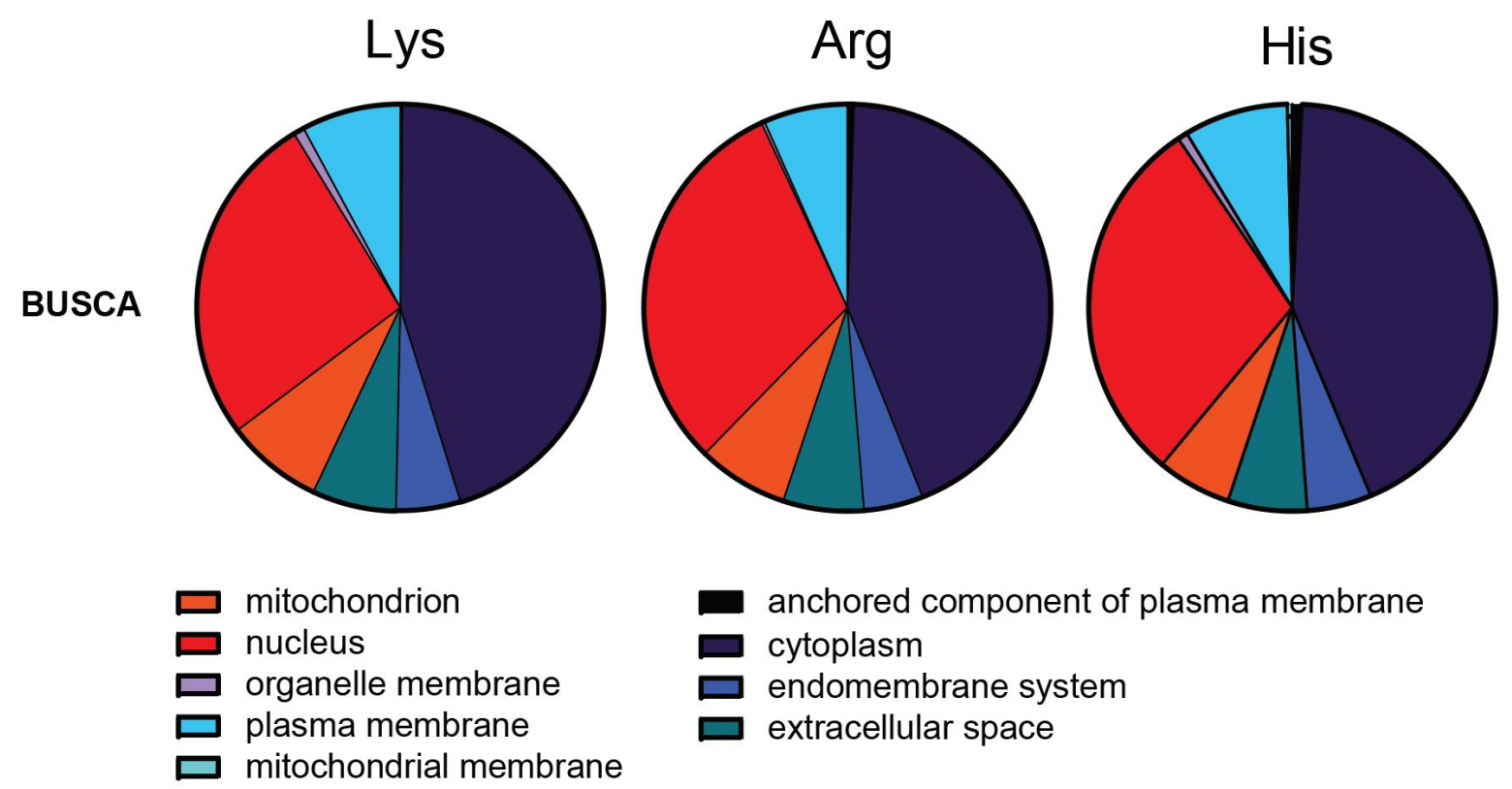

anchored component of plasma membrane

$\square$ cytoplasm

$\square$ endomembrane system

$\square$ extracellular space

684 Supplementary Figure S5. Methylated proteins are distributed throughout the cell.

685 The subcellular localization of proteins methylated in HeLa cells. Pie charts representing the relative 686 predicted localization based on the BUSCA prediction ${ }^{19}$. 


\section{Supplementary Figure S6}

\section{A}

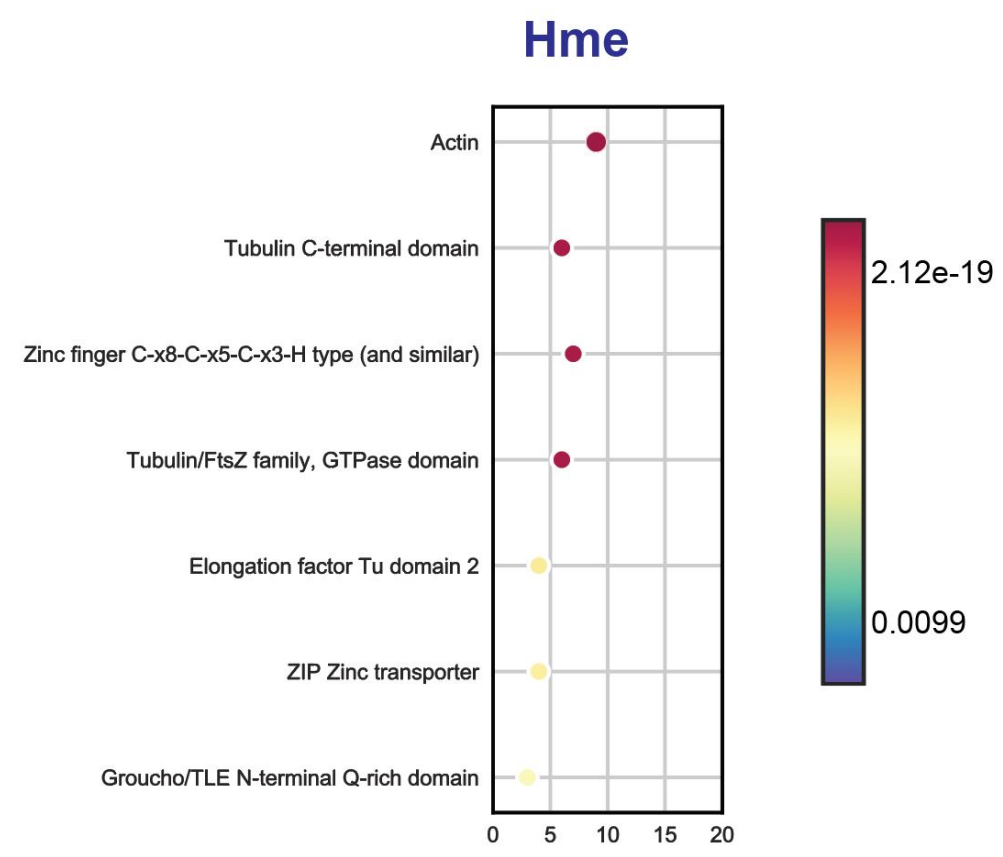

\section{B}

RNA recognition motif. (a.k.a. RRM, RBD, or RNP domain)

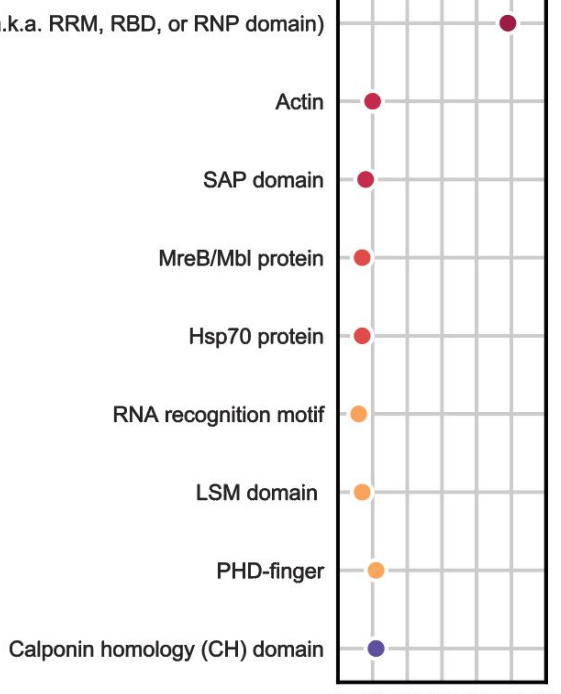

Rme

0102030405060
C

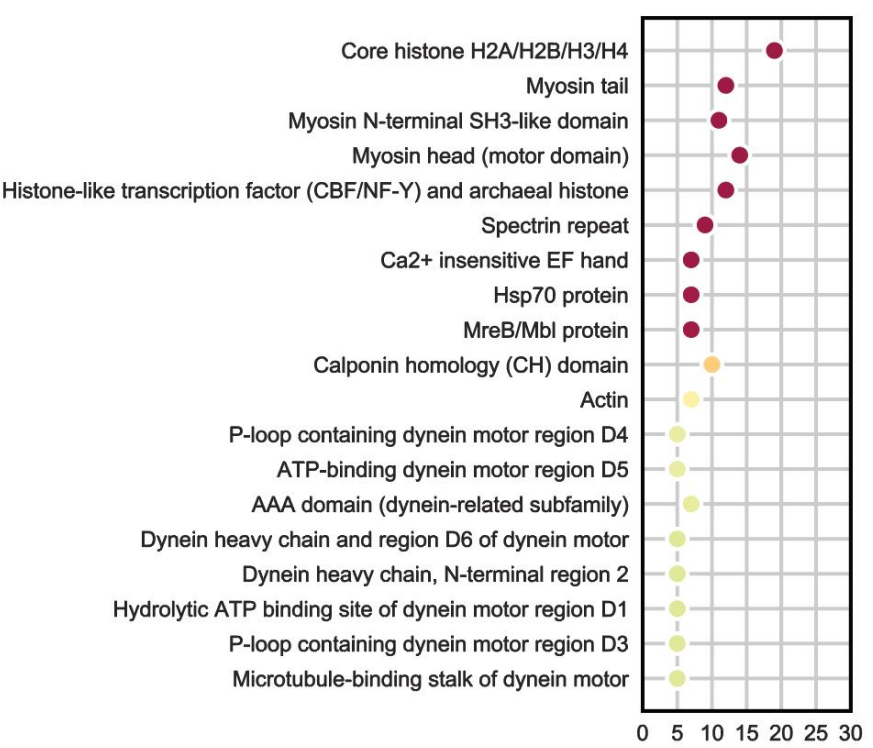

\section{Supplementary Figure S6. Domain enrichment analysis of proteins containing a methylation site.}

689 Functional enrichment analysis performed on methylated proteins based using the Pfam database, 690 categories considered significant ( $p$-value $<0.01$ ) were adjusted using the Benjamini-Hochberg method.

691 The total number of proteins having a significant enrichment in each category is shown on the $x$-axis. 


\section{Supplementary Figure S7}

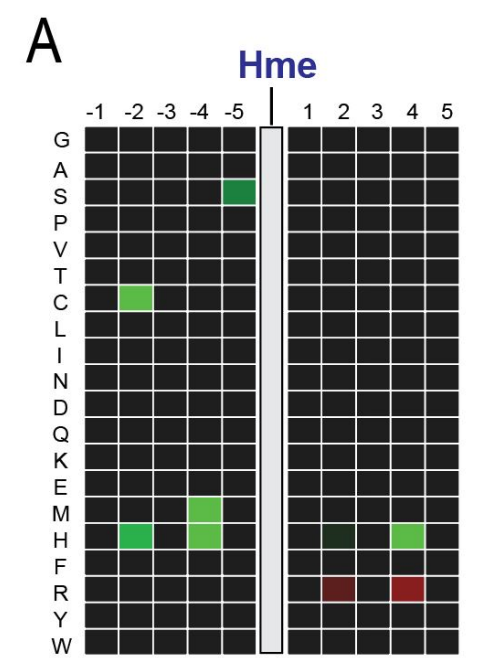

B

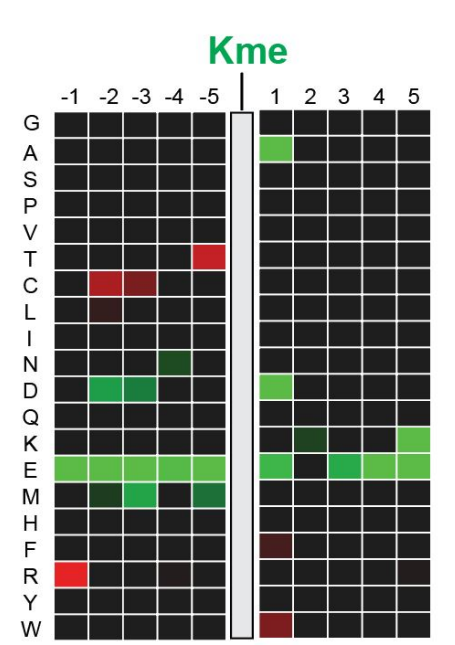

C
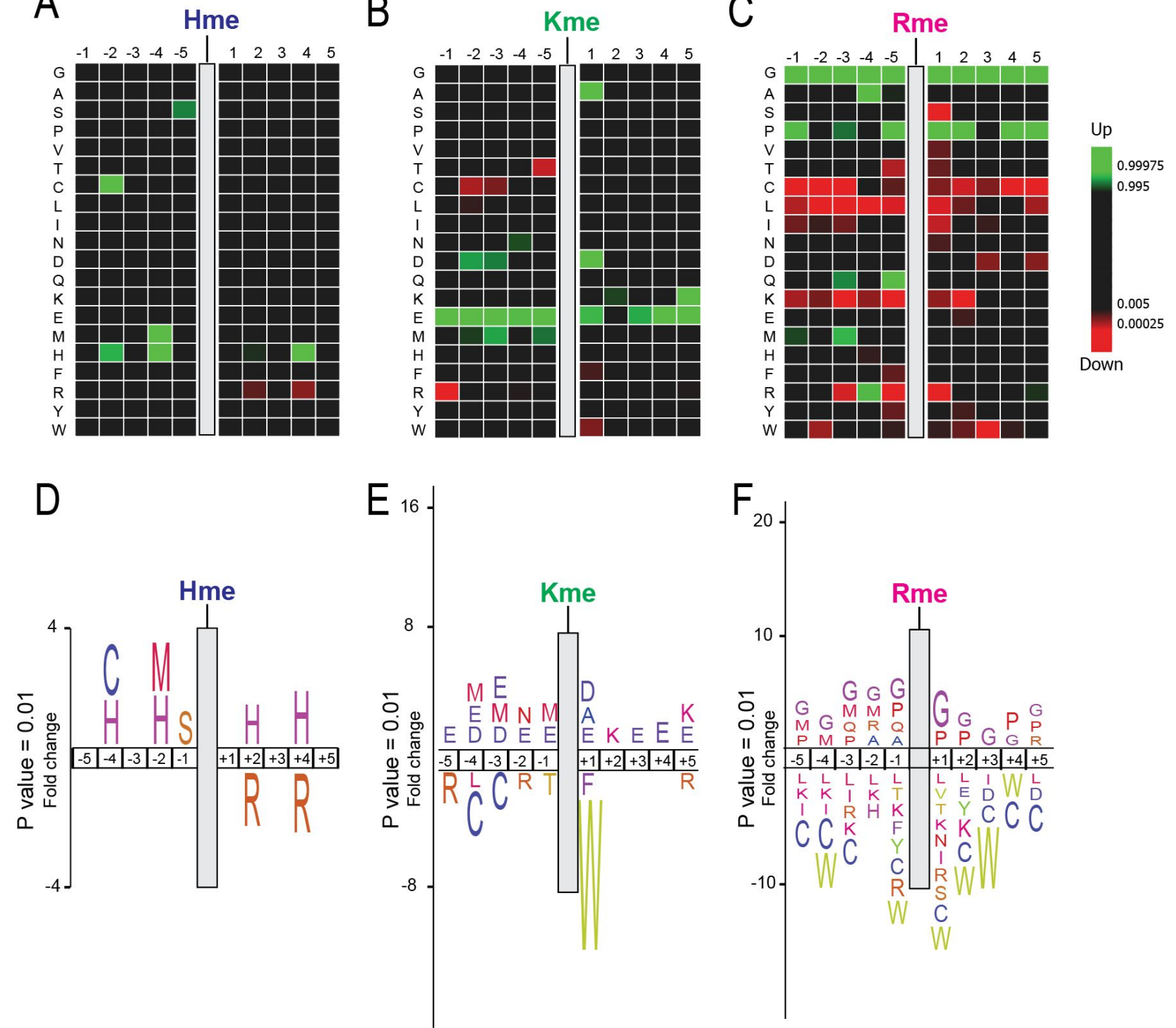

694 (A-C) Methylome heat maps. Heat maps illustrating over- or under-representation of amino acids in 695 the 5 positions up- and down-stream of identified (A) Hme, (B) Kme, and (C) Rme sites are shown. 696 The enrichment is based on using the H. Sapiens precompiled Swiss-Prot composition as reference.

697 (D-F) Methylome sequence logos. Logo representations of the heat maps in (A-C) are shown. 